\title{
A Hierarchical Cluster System Based on Horton-Strahler Rules for River Networks
}

\author{
F.P. da Costa
}

Instituto Superior Técnico, Dep. de Matemática

Av. Rovisco Pais 1, P-1049-001 Lisboa, Portugal

M. Grinfeld

University of Strathclyde, Dep. of Mathematics

26 Richmond Street, Glasgow G1 1XH, UK

\author{
J.A.D. Wattis \\ University of Nottingham, School of Mathematical Sciences \\ University Park, Nottingham NG7 2RD, UK
}

\begin{abstract}
We consider a cluster system in which each cluster is characterized by two parameters: an "order" $i$, following Horton-Strahler's rules, and a "mass" $j$ following the usual additive rule. Denoting by $c_{i, j}(t)$ the concentration of clusters of order $i$ and mass $j$ at time $t$, we derive a coagulationlike ordinary differential system for the time dynamics of these clusters. Results about existence and the behaviour of solutions as $t \rightarrow \infty$ are obtained, in particular we prove that $c_{i, j}(t) \rightarrow 0$ and $N_{i}(c(t)) \rightarrow 0$ as $t \rightarrow \infty$, where the functional $N_{i}(\cdot)$ measures the total amount of clusters of a given fixed order $i$. Exact and approximate equations for the time evolution of these functionals are derived. We also present numerical results that suggest the existence of self-similar solutions to these approximate equations and discuss its possible relevance for an interpretation of Horton's law of river numbers.
\end{abstract}

Keywords: Coagulation equations, cluster dynamics, Horton-Strahler rules. 


\section{Introduction}

In recent years a great deal of effort has been dedicated to the understanding of the differential equations modelling the kinetics of cluster growth. In the majority of these studies, a cluster of particles is identified by a positive number, either integer or real, denoting the "size", or "mass", of the cluster, as measured in a convenient scale (see, eg [1], and references therein.)

There are other cases, however, where a single parameter is not enough to describe the cluster population.

One such situation occurs in polymerization studies when two different chemical species, $\mathcal{A}$ and $\mathcal{B}$ say, constitute the building blocks (monomers) for the clusters (polymers) and so, in order to characterize a given cluster one needs to know, not only the total mass of the cluster, but also the amount of $\mathcal{A}$ monomers used in its formaton. In the spatially homogeneous situation, this implies the phase variables to be nonnegative valued functions $c_{i, j}(t)$, where, for instance, $i$ could denote the number of $\mathcal{A}$ monomers and $j$ that of $\mathcal{B}$ monomers in the cluster made up of $i+j$ monomeric units, and $c_{i, j}(t)$ would denote the concentration of that cluster as a function of time, [2].

Another situation is when one considers clusters constructed with only one type of monomeric units, as in the usual coagulating systems, but the clusters thus formed can have a number of different competitive morphologies, so that the description of the clusters involve a phase variable $c_{p, q}(t)$ with $q$ denoting the mass and $p$ the morphological type of the cluster, [3].

As far as we are aware, the only mathematically rigorous analysis of these kind of multi-parametrized cluster systems has been done for the two-component Becker-Döring equation in [4].

In the present paper we shall consider a cluster system for which the clusters are identified by a "mass" $j$ and an "order" $i$. The motivation for this second variable, and for the functional rules it obeys, arises from geomorphological studies that we shall now briefly describe.

\subsection{The Horton-Strahler Rules}

In the 1940s, attempts by geologists to quantify the morphological descriptions of river networks let to the introduction of a number of parameters intended to reflect, in a quantitative way, the intuitive notions of main and affluent channels in a river network. The first such study was the seminal article of Robert Horton [5]. In it, Horton introduced the concept of order of a river stream and the operational rules allowing to compute the orders of all the streams in a given river network. The original idea of Horton had the drawback of requiring sucessive renumbering of river orders already computed, and so it was not convenient from a computational viewpoint. This problem was soon overcomed by Arthur Strahler, with a small but important modification of Horton's ideas, [6]. The resulting notion of order, and rules for its computation, commonly refered to as the Horton-Strahler rules, are presented next. 
A river network (having neither islands, multiple branching points nor deltas) can be associated with a binary rooted tree, river streams corresponding to edges of the tree. The order is the function defined on the edges of the tree, with values in $\mathbb{N}_{+}$, and given by the following rules:

(i) edges connected to leaves have order 1 .

(ii) if two edges of orders $i$ and $j$ concur at a node, the edge downstream has order $i \vee j$ if $i \neq j$, or $j+1$ if $i=j$.

We shall call (i)-(ii) the Horton-Strahler rules (see Figure 1).

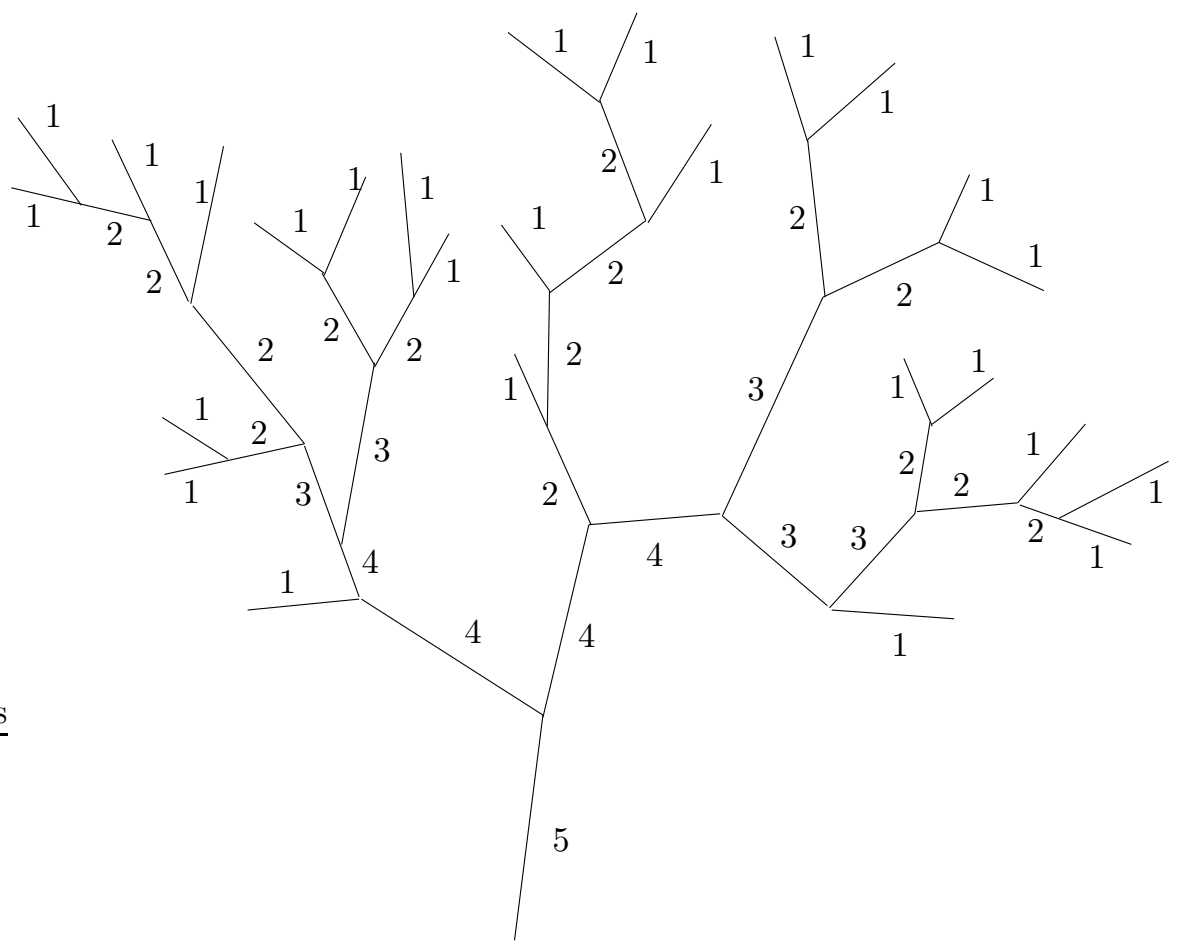

Figure 1: Illustration of the Horton-Strahler rules in a given tree

A number of empirical relationships about river networks was first observed by Horton himself in [5]. One is the commonly called Horton's law of stream numbers, stating that, if $n_{i}$ is the number of river segments with Horton-Strahler order $i$ in a given network, then $n_{i}=R_{b} n_{i+1}$, where $R_{b}$ is a constant independent of $i$ (called the bifurcation ratio of the network) usually between 3 and 5 for river networks in Continental US. Similar empirical laws for stream lengths and drainage areas also hold, [7, 8].

It is interesting to note that Horton-Strahler rules play a significant role in several branches of science, not only in geomorphology but also in theoretical 
computer science (where it is called the register function) [9, 10], computer generated images, and structures in molecular biology (see [10] and references therein.)

\subsection{The Cluster System}

Recently, in [11], Horton-Strahler rules have also been applied to cluster kinetic modelling of forest fires dynamics. In that paper, the authors made a very interesting attempt to obtain a cluster model for which each given cluster is characterized by its mass and also by an Horton-Strahler order (they call it "rank"). A wide range of interesting questions, such as Horton's type laws, self-similarity, and scaling were addressed. However, results in [11] are not rigorously deduced, and even the rigorous justification of the kinetic equations used seems very problematic, at least in the context of the usual mass action law assumptions.

In the present article we propose a system of ordinary differential equations derived from the application of the mass action law of chemical kinetics to a cluster system for which the concentration of each cluster is represented by a real function $c_{i, j}(t)$ indexed by the order $i$ and the mass $j$. Our original goal was to turn rigorous the formal analysis of [11]. However, it turned out that the kinetic equations obtained for the system considered in [11] where far too hard to analyse than expected: in fact they were a kind of Becker-Döring type equations, with constant input of monomers, and involving three-body reactions. Even for the usual Becker-Döring equations with input of monomers, the long time behaviour of solutions is not completely understood, [12], and so it seemed advisable to start with a simpler case than that studied in [11], namely we consider a cluster system with no inputs or outputs and involving only two-cluster coagulations without Becker-Döring type restrictions. These assumptions result in a coagulation-like system for the phase variables $c_{i, j}(t)$ that we shall now present in detail. As pointed out above, in $c_{i, j}(t)$ the first subscript, $i$, denotes the order of the cluster, and the second, $j$, its mass. Masses satisfy the usual local mass conservation: a cluster of mass $j$ reacts with a cluster of mass $m$ to produce a cluster of mass $j+m$. For the orders, the Horton-Strahler rules are assumed to hold: a cluster of order $i$ reacts with a cluster of order $k$ to result in a cluster or order $\bigvee(i, k,(i \wedge k)+1)$. We assume the order of a cluster is never larger than its mass, and also that there exists only one type of clusters of order 1 , which have mass also normalized to 1 . Representing a cluster of order $p$ and mass $q$ by $(p, q)$, the cluster reactions assumed in this work can be schematically represented using the notation normaly used in chemical kinetics:

$$
(i, j)+(k, m) \rightarrow(\bigvee(i, k,(i \wedge k)+1), j+m)
$$

with $j \geq i$ and $k \geq m$.

In order to make the derivation of the rate equations as transparent as possible, we re-write (1) in the following more explicit form, which correspond to (i) and (ii) above 
(H1) $(i, j)+(i, m) \rightarrow(i+1, j+m)$

(H2) $(i, j)+(k, m) \rightarrow(i \vee k, j+m) \quad(i \neq k)$.

The general kinetic coefficients for these reactions between clusters can, quite clearly, depend on both the orders and the masses of the intervening clusters. With the objective of simplifying the analysis in this study, we will only consider the case where only the order comes into play, and furthermore the kinetics is assumed to be of the product type, namely, the kinetic coefficient for the reaction written in (1) is $\ell_{j} \ell_{m}$, for some nonnegative sequence $\left(\ell_{j}\right)$. All results in Sections 2,3 and 4 below should still hold under more general kinetic coefficients.

This assumption on the kinetic coefficients means that the rate of increase in the concentration of the $(\bigvee(i, k,(i \wedge k)+1), j+m)$-cluster due to the reaction represented in (1) is, by the mass action law, given by

$$
\ell_{j} \ell_{m} c_{i, j}(t) c_{k, m}(t)
$$

In the spirit of previous works on cluster systems, we will not impose any upper limit on the cluster's masses or order. Remembering the restrictions that in clusters $(i, j)$ we always have $j \geq i$ for all $i \geq 2$ and that $(1,1)$ is the only cluster of order 1 , the infinite system of ordinary differential equations modelling the time evolution of the concentrations of the various $(i, j)$-clusters is the following:

$$
\begin{aligned}
& \dot{c}_{1,1}=-\underbrace{2 \ell_{1}^{2} c_{1,1}^{2}}_{\text {(due to (H1)) }}-\underbrace{\ell_{1} c_{1,1} \sum_{i=2}^{\infty} \sum_{j=i}^{\infty} \ell_{i} c_{i, j}}_{\text {(due to (H2)) }} \\
& \dot{c}_{i, j}=\underbrace{\ell_{1}^{2} c_{1,1}^{2} \delta_{i=2} \delta_{j=2}+\sum_{m=\left\lfloor\frac{j+1}{2}\right\rfloor}^{j-i+1} \ell_{i-1}^{2} c_{i-1, m} c_{i-1, j-m} \delta_{j \geq 2 i-2} \delta_{i \geq 3}}_{\text {(increase due to (H1)) }} \\
& +\underbrace{\ell_{1} c_{1,1} \ell_{i} c_{i, j-1} \delta_{j \geq i+1}+\sum_{m=i}^{j-2} \sum_{k=2}^{(i-1) \wedge(j-m)} \ell_{i} \ell_{k} c_{i, m} c_{k, j-m} \delta_{j \geq i+2} \delta_{i \geq 3}}_{\text {(increase due to }(\mathrm{H} 2))} \\
& -\underbrace{\ell_{i} c_{i, j} \sum_{\substack{m=i \\
m \neq j}}^{\infty} \ell_{i} c_{i, m}-2 \ell_{i}^{2} c_{i, j}^{2}}_{\text {(decrease due to (H1)) }}-\underbrace{\ell_{1} c_{1,1} \ell_{i} c_{i, j}-\sum_{\substack{k=2 \\
k \neq i}}^{\infty} \sum_{m=k}^{\infty} \ell_{i} \ell_{k} c_{i, j} c_{k, m}}_{\text {(decrease due to (H2)) }}
\end{aligned}
$$

where we use the notation $\lfloor x\rfloor$ for the integer part of $x$, and

$$
\delta_{\mathcal{P}}:= \begin{cases}1 & \text { if } \mathcal{P} \text { is true } \\ 0 & \text { if } \mathcal{P} \text { is false. }\end{cases}
$$


Note that the terms in the $c_{i, j}$ equation corresponding to the decrease due to (H1) (first two terms in (5)) can be written in the simpler form

$$
-\ell_{i} c_{i, j} \sum_{m=i}^{\infty} \ell_{i} c_{i, m}-\ell_{i}^{2} c_{i, j}^{2}
$$

Since the second subscript in $c_{i, j}$ denotes the mass of the cluster, the quantity

$$
\rho(c):=c_{1,1}+\sum_{i=2}^{\infty} \sum_{j=i}^{\infty} j c_{i, j}
$$

can be naturally interpreted as the total density of the system.

Another quantity of interest is the total amount of clusters of a given order $i$. We shall denote it by $N_{i}(\cdot)$ and define it by

$$
N_{1}(c):=c_{1,1}, \quad \text { and } \quad N_{i}(c):=\sum_{j=i}^{\infty} c_{i, j} \text { if } i \geq 2
$$

The time evolution of $N_{i}(c(t))$ is something whose study is of obvious interest. Already in the context of river network problems, the number of rivers of a given order, $n_{i}$, was a quantity of basic interest, being the object of one of Horton's laws. In the context of cluster systems, these quantities $N_{i}(c)$ can be interpreted as a kind of mesoscopic variables, describing the system at an intermediate scale, between the microscopic description provided by the phase variables $c_{i, j}(t)$ and the macroscopic quantities, such as the total density $\rho(c)$, or the total number of clusters $\mathcal{N}_{\infty}(c):=\sum_{i=1}^{\infty} N_{i}(c)$. Hence, in this sense, they can be seen as a natural coarser description of the system, with some similarities to other coarse-graining procedures recently proposed in the study of cluster systems, [2, 13].

Associated with (2)-(5), we can consider a density conserving and finite dimensional system, by considering an appropriately chosen truncation of the infinite system, akin to what is normaly done for the usual coagulation equations, [14]. This finite dimensional system will be presented and studied in Section 2.

We now describe the contents of the paper:

In Section 2 we introduce and study a truncated finite dimensional system of ordinary differential equations obtained from (2)-(5). We prove some auxiliary results that will be of use in later sections, and in particular the algebraic manipulations in the proof of Proposition 3 are presented in some detail since they are also of use in later sections.

In Section 3 we consider the problem of existence and uniqueness of solutions to (2)-(5). Results in that Section are not proved under the weakest possible assumptions. We believe that a careful analysis analogous to what was done in [15] for the Smoluchowski equations can also be applied to the present case, leading to much sharper existence results. As for the uniqueness, even in the case of the Smoluchowki coagulation equations, current existence results seem 
to be far from optimal: here we briefly outline the steps of the proof, which essentially follows the idea used, for instance, in [16] and [17].

Section 4 contains a rigorous study of the long time behaviour of solutions to (2)-(5) with positive kinetic coeficients. We prove two results: first that all components $c_{i, j}(t)$ of every solution converge to zero as $t \rightarrow+\infty$; second, that the total amount of clusters of any given fixed order $i, N_{i}(c(t))$, converge to zero as $t \rightarrow+\infty$. Clearly, since we are in presence of a coagulation-type system (albeit a much more cumbersome one than usual), these results are not really surprising; however the proofs are somewhat more involved than in the Smoluchowski equation [18], and, in particular, the proof of the result concerning $c_{i, j}(t)$ requires the use of two families of Lyapunov functionals. Concerning the behaviour of $N_{i}(c(t))$, a differential inequality is obtained for its time evolution and it is then exploited to get the desired result.

Finally, in Section 5 we try to gain a better understanding of the dynamics of the mesoscopic variables $N_{i}(c(t))$. The time evolution of these quantities is not governed by a closed system of ordinary differential equations; however, the (closed) set of differential inequalities obtained in Section 4 can be used to obtain approximate closed differential systems that are more amenable to analysis. In Section 5 we work at some lenght in the analysis of one such system and refer more briefly to another one. Part of the results, concerning convergence to equilibria and the unimodality of solutions are proved rigorously, but to the more demanding problem of self-similar behaviour of solutions we were unable to obtain rigorous results, and we instead present some suggestive computations and formal arguments whose rigorous exploitation we hope to return to in a later work.

\subsection{Some Preliminaries}

Since the phase variables $c_{i, j}(t)$ are indexed by two positive integers it is convenient to define the following subsets of $\mathbb{N}_{+}^{2}$ for later use:

$$
\begin{array}{ll}
\Xi & :=\{(1,1)\} \cup\{(i, j): j \geq i \geq 2\} \\
\Xi_{N} & :=\Xi \cap[1, N]^{2} .
\end{array}
$$

For the study of (2)-(5) we need to introduce the following subspaces of $\ell_{1}$ : for $p$ and $q$ two nonnegative real numbers, let

$$
X_{p, q}:=\left\{c=\left(c_{i, j}\right):\|c\|_{p, q}<\infty\right\}
$$

where $c_{i, j}: \Xi \rightarrow \mathbb{R}$, and

$$
\|c\|_{p, q}:=\sum_{(i, j) \in \Xi} i^{p} j^{q}\left|c_{i, j}\right|=\left|c_{1,1}\right|+\sum_{i=2}^{\infty} \sum_{j=i}^{\infty} i^{p} j^{q}\left|c_{i, j}\right| .
$$

It is not difficult to check that $\|\cdot\|_{p, q}$ is a norm in $X_{p, q}$ and $\left(X_{p, q},\|\cdot\|_{p, q}\right)$ is a Banach space. We shall denote by $X_{p, q}^{+}$the nonnegative cone of $X_{p, q}$. Let us 
consider the following partial order in $\mathbb{N}_{+}^{2}$ :

$$
(p, q) \prec(r, s) \Longleftrightarrow p \leq r \wedge q \leq s \wedge(p, q) \neq(r, s) .
$$

With this notation we can now state the following natural embedding and interpolation inequalities between the spaces $X_{p, q}$ :

Proposition 1 For all $(p, q) \prec(r, s)$ it holds $X_{r, s} \hookrightarrow X_{p, q}$ with dense and compact embedding, and $\|c\|_{p, q} \leq\|c\|_{r, s}$

Proposition 2 (i) Let $\left(\alpha_{1}, \beta\right) \prec\left(\alpha_{2}, \beta\right) \prec\left(\alpha_{3}, \beta\right)$ and $c \in X_{\alpha_{3}, \beta}$. Then

$$
\|c\|_{\alpha_{2}, \beta}^{\alpha_{3}-\alpha_{1}} \leq\|c\|_{\alpha_{1}, \beta}^{\alpha_{3}-\alpha_{2}}\|c\|_{\alpha_{3}, \beta}^{\alpha_{2}-\alpha_{1}}
$$

(ii) Let $\left(\alpha, \beta_{1}\right) \prec\left(\alpha, \beta_{2}\right) \prec\left(\alpha, \beta_{3}\right)$ and $c \in X_{\alpha, \beta_{3}}$. Then

$$
\|c\|_{\alpha, \beta_{2}}^{\beta_{3}-\beta_{1}} \leq\|c\|_{\alpha, \beta_{1}}^{\beta_{3}-\beta_{2}}\|c\|_{\alpha, \beta_{3}}^{\beta_{2}-\beta_{1}} .
$$

The proofs are entirely similar to those of the corresponding results for the spaces used in the study of the usual coagulation-fragmentation equations, and presented in Propositions 2.1 and 2.2 of [17]; we shall omit them here.

We adopt for the definition of solution a notion analogous to the one used in [16]. Writing system (2)-(5) as

$$
\dot{c}_{i, j}=\sum_{k} F_{k, i, j}(c),
$$

we will use the following

Definition 1 Let $T \in(0, T]$. A solution $c=\left(c_{i, j}\right)$ of (2)-(5) with initial condition $c^{0}=\left(c_{i, j}^{0}\right) \in\left(X_{0,1} \cap X_{1,0}\right)^{+}$is a function $c:[0, T) \rightarrow\left(X_{0,1} \cap X_{1,0}\right)^{+}$such that

(i) $\sup _{t \in[0 . T)}\left(\|c(t)\|_{1,0}+\|c(t)\|_{0,1}\right)<\infty$, and $\forall(i, j) \in \Xi, c_{i, j} \in \mathcal{C}([0, T))$.

(ii) $\forall(i, j) \in \Xi, \forall t \in[0, T), \int_{0}^{t} F_{k, i, j}(c(s)) d s<\infty$.

(iii) $\forall(i, j) \in \Xi, \forall t \in[0, T), \quad c_{i, j}(t)=c_{i, j}^{0}+\sum_{k} \int_{0}^{t} F_{k, i, j}(c(s)) d s$.

\section{The Truncated System}

In order to prove results for the infinite system we shall need to consider a convenient finite dimensional truncation. In this work we shall use a truncated system that corresponds to just taking into account the clusters with mass $j \leq N$ and not allowing a reaction between two clusters to occur if the resulting cluster would violate this condition. Hence, with these rules, the truncated system for 
the functions $c^{N}=\left(c_{i, j}^{N}\right)$ can be written as follows, with the terms in the righthand side in the same order as in equations (2)-(5) to make comparisons easier,

$$
\left.\begin{array}{rl}
\dot{c}_{1,1}^{N}= & -2 \ell_{1}^{2}\left(c_{1,1}^{N}\right)^{2}-\ell_{1} c_{1,1}^{N} \sum_{i=2}^{N-1} \sum_{j=i}^{N-1} \ell_{i} c_{i, j}^{N} \\
\dot{c}_{i, j}^{N}= & \ell_{1}^{2}\left(c_{1,1}^{N}\right)^{2} \delta_{i=2} \delta_{j=2} \\
& +\sum_{m=\left\lfloor\frac{j+1}{2}\right\rfloor}^{j-i+1} \ell_{i-1}^{2} c_{i-1, m}^{N} c_{i-1, j-m}^{N} \delta_{2 i-2 \leq j \leq N} \delta_{3 \leq i \leq 1+\left\lfloor\frac{N}{2}\right\rfloor} \\
& +\ell_{1} c_{1,1}^{N} \ell_{i} c_{i, j-1}^{N} \delta_{3 \leq i+1 \leq j \leq N} \\
& +\sum_{m=i}^{j-2} \sum_{k=2}^{N i-1) \wedge(j-m)} \ell_{i} \ell_{k} c_{i, m}^{N} c_{k, j-m}^{N} \delta_{i+2 \leq j \leq N} \delta_{3 \leq i \leq N-2} \\
& -\ell_{i} c_{i, j}^{N} \sum_{m=i}^{N-j} \ell_{i} c_{i, m}^{N} \delta_{i \leq j \leq N-i} \delta_{2 \leq i \leq\left\lfloor\frac{N}{2}\right\rfloor}-\ell_{i}^{2}\left(c_{i, j}^{N}\right)^{2} \delta_{2 \leq i \leq j \leq\left\lfloor\frac{N}{2}\right\rfloor} \\
& -\ell_{1} c_{1,1}^{N} \ell_{i} c_{i, j}^{N} \delta_{2 \leq i \leq j \leq N-1}-\sum_{\substack{k=2 \\
k \neq i}}^{N-j} \sum_{m=k}^{N-j} \ell_{i} \ell_{k} c_{i, j}^{N} c_{k, m}^{N} \delta_{2 \leq i \leq j \leq N-2}
\end{array}\right\}
$$

The existence and uniqueness of local solutions to Cauchy problems for (8)-(9) is guaranteed by the standard Picard-Lindelöf theorem for ordinary differential equations. The nonnegativity of solutions with nonnegative initial data can be easily proved by the method of adding $\varepsilon>0$ to the right hand side of (8)-(9) as in [19, Lemma 2.1].

Defining the truncated density in the natural way, namely,

$$
\rho^{N}(c):=c_{1,1}+\sum_{i=2}^{N} \sum_{j=i}^{N} j c_{i, j}
$$

we shall prove that $\rho^{N}\left(c^{N}\right)$ is time invariant. In fact, this is a consequence of the following more general result:

Proposition 3 Let $T>0$. Let $c^{N}=\left(c_{i, j}^{N}\right)$ be any solution to (8)-(9) in $[0, T)$. Consider $c^{N}$ as an element of $\mathbb{R}^{\Xi}$ by defining $c_{i, j}^{N}=0$ for all $(i, j) \notin \Xi_{N}$. Then, 
in $(0, T)$, the following holds true for any $g=\left(g_{i, j}\right) \in \mathbb{R}^{\Xi}$,

$$
\begin{aligned}
\frac{d}{d t} \sum_{(i, j) \in \Xi} g_{i, j} c^{N}= \\
\quad\left(g_{2,2}-2 g_{1,1}\right) \ell_{1}^{2}\left(c_{1,1}^{N}\right)^{2} \\
+\ell_{1} c_{1,1}^{N} \sum_{i=2}^{N-1} \sum_{j=i}^{N-1}\left(g_{i, j+1}-g_{i, j}-g_{1,1}\right) \ell_{i} c_{i, j}^{N} \\
+\sum_{i=2}^{\left\lfloor\frac{N}{2}\right\rfloor} \sum_{j=i}^{\left\lfloor\frac{N}{2}\right\rfloor} \sum_{m=j}^{N-j}\left(g_{i+1, j+m}-g_{i, j}-g_{i, m}\right) \ell_{i}^{2} c_{i, j}^{N} c_{i, m}^{N} \\
+\sum_{i=2}^{\left\lfloor\frac{N-1}{2}\right\rfloor} \sum_{k=i+1}^{N-i} \sum_{j=i}^{N-k} \sum_{m=k}^{N-j}\left(g_{k, j+m}-g_{i, j}-g_{k, m}\right) \ell_{i} \ell_{k} c_{i, j}^{N} c_{k, m}^{N}
\end{aligned}
$$

Proof: From the truncated system (8)-(9), after grouping the terms conveniently, we obtain,

$$
\begin{aligned}
\frac{d}{d t} \sum_{(i, j) \in \Xi} g_{i, j} c^{N}= & g_{1,1} \dot{c}_{1,1}^{N}+\sum_{i=2}^{N} \sum_{j=i}^{N} g_{i, j} \dot{c}_{i, j}^{N} \\
= & \ell_{1}^{2}\left(c_{1,1}^{N}\right)^{2}\left(g_{2,2}-2 g_{1,1}\right) \\
& +\ell_{1} c_{1,1}^{N}\left(-\sum_{i=2}^{N-1} \sum_{j=i}^{N-1} g_{1,1} \ell_{i} c_{i, j}^{N}+\sum_{i=2}^{N-1} \sum_{j=i+1}^{N} g_{i, j} \ell_{i} c_{i, j-1}^{N}\right. \\
& \\
& \left.+\sum_{i=2}^{N-1} \sum_{j=i}^{N-1} g_{i, j} \ell_{i} c_{i, j}^{N}\right)
\end{aligned}
$$


where

$$
\begin{aligned}
Q_{g}^{N}\left(c^{N}\right)= & \sum_{i=3}^{\left\lfloor\frac{N}{2}\right\rfloor+1} \sum_{j=2 i-2}^{N} \sum_{m=\left\lfloor\frac{j+1}{2}\right\rfloor}^{j-i+1} g_{i, j} \ell_{i-1}^{2} c_{i-1, m}^{N} c_{i-1, j-m}^{N} \\
& +\sum_{i=3}^{N-2} \sum_{j=i+2}^{N} \sum_{m=i}^{j-2} \sum_{k=2}^{(i-1) \wedge(j-m)} g_{i, j} \ell_{i} \ell_{k} c_{i, m}^{N} c_{k, j-m}^{N} \\
& -\sum_{i=2}^{\left\lfloor\frac{N}{2}\right\rfloor} \sum_{j=i}^{N-i} \sum_{m=i}^{N-j} g_{i, j} \ell_{i}^{2} c_{i, j}^{N} c_{i, m}^{N} \\
& -\sum_{i=2}^{\left\lfloor\frac{N}{2}\right\rfloor} \sum_{j=i}^{\left\lfloor\frac{N}{2}\right\rfloor} g_{i, j} \ell_{i}^{2}\left(c_{i, j}^{N}\right)^{2} \\
& -\sum_{i=2}^{N-2} \sum_{j=i}^{N-2} \sum_{\substack{k=2 \\
k \neq i}}^{N-j} \sum_{m=k}^{N-j} g_{i, j} \ell_{i} \ell_{k} c_{i, j}^{N} c_{k, m}^{N}
\end{aligned}
$$

if $N \geq 4$ and $Q_{g}^{N}\left(c^{N}\right)=0$ otherwise. (We define a sum to be zero if its upper index is smaller than its lower one.) Expression (15) is exactly the same as (11). To get (12) we simply relabel $j-1 \mapsto j$ in the second double sum in (16). The expressions (13) and (14) originate from (17) by a much more delicate algebraic manipulation, that we now briefly describe.

For (18) we start by changing the order of summation from $\sum_{j} \sum_{m}$ to $\sum_{m} \sum_{j}$ and relabeling $j-m \mapsto j$ and $i-1 \mapsto i$ to obtain

$$
\begin{aligned}
\sum_{i=3}^{\left\lfloor\frac{N}{2}\right\rfloor+1} \sum_{j=2 i-2}^{N} \sum_{m=\left\lfloor\frac{\lfloor+1}{2}\right\rfloor}^{j-i+1} g_{i, j} \ell_{i-1}^{2} c_{i-1, m}^{N} c_{i-1, j-m}^{N}= \\
=\sum_{i=2}^{\left\lfloor\frac{N}{2}\right\rfloor} \sum_{m=\left\lfloor\frac{N+1}{2}\right\rfloor}^{N-i} \sum_{j=i}^{N-m} g_{i+1, j+m} \ell_{i}^{2} c_{i, j}^{N} c_{i, m}^{N}+ \\
+\sum_{i=2}^{\left\lfloor\frac{N-1}{2}\right\rfloor} \sum_{m=i}^{\left\lfloor\frac{N-1}{2}\right\rfloor} \sum_{j=i}^{m} g_{i+1, j+m} \ell_{i}^{2} c_{i, j}^{N} c_{i, m}^{N}
\end{aligned}
$$

and, changing the order of summation in the last multiple sum from $\sum_{m} \sum_{j}$ to 
$\sum_{j} \sum_{m}$, expression (18) becomes

$$
\begin{aligned}
& \sum_{i=2}^{\left\lfloor\frac{N}{2}\right\rfloor} \sum_{m=\left\lfloor\frac{N+1}{2}\right\rfloor}^{N-i} \sum_{j=i}^{N-m} g_{i+1, j+m} \ell_{i}^{2} c_{i, j}^{N} c_{i, m}^{N}+ \\
& \quad+\sum_{i=2}^{\left\lfloor\frac{N-1}{2}\right\rfloor} \sum_{j=i}^{\left\lfloor\frac{N-1}{2}\right\rfloor} \sum_{m=j}^{\left\lfloor\frac{N-1}{2}\right\rfloor} g_{i+1, j+m} \ell_{i}^{2} c_{i, j}^{N} c_{i, m}^{N}
\end{aligned}
$$

From Figure 2, where, in the $(j, m)$-space, $S_{1}$ and $S_{2}$ represent the summation region of the first and the second multiple sums in (23) respectively, we can write (23), and thus (18), as being equal to

$$
\sum_{i=2}^{\left\lfloor\frac{N}{2}\right\rfloor} \sum_{j=i}^{\left\lfloor\frac{N}{2}\right\rfloor} \sum_{m=j}^{N-j} g_{i+1, j+m} \ell_{i}^{2} c_{i, j}^{N} c_{i, m}^{N}
$$

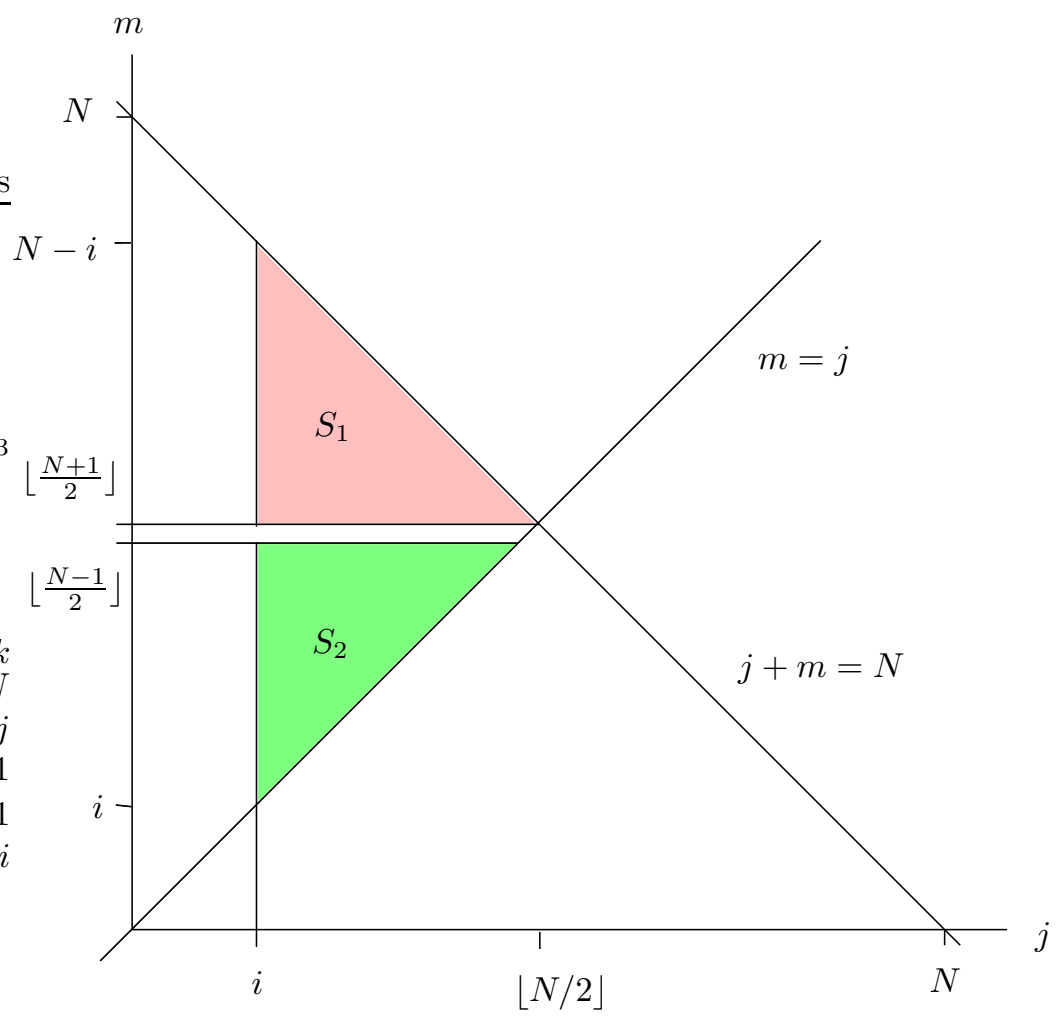

Figure 2: Summation regions in expression (23).

For the term (19) in the expression of $Q_{g}^{N}$ one can, in succession, change the order of summation $\sum_{j} \sum_{m}$ to $\sum_{m} \sum_{j}$, rename $j-m \mapsto j$ and change notation 
$(m, j) \mapsto(j, m)$ to obtain

$$
\begin{gathered}
\sum_{i=3}^{N-2} \sum_{j=i+2}^{N} \sum_{m=i}^{j-2} \sum_{k=2}^{(i-1) \wedge(j-m)} g_{i, j} \ell_{i} \ell_{k} c_{i, m}^{N} c_{k, j-m}^{N}= \\
=\sum_{i=3}^{N-2} \sum_{j=i}^{N-2} \sum_{m=2}^{N-j} \sum_{k=2}^{(i-1) \wedge m} g_{i, j+m} \ell_{i} \ell_{k} c_{i, j}^{N} c_{k, m}^{N}
\end{gathered}
$$

and considering the triangular pyramid defined by the region of summation in the space $(i, j, m)$, and, considering separately its parts with $m>i-1$ and with $m \leq i-1$ one can write the above expression as

$$
\begin{aligned}
& \sum_{m=2}^{\left\lfloor\frac{N-1}{2}\right\rfloor} \sum_{i=m+1}^{N-m} \sum_{j=i}^{N-m} \sum_{k=2}^{m} g_{i, j+m} \ell_{i} \ell_{k} c_{i, j}^{N} c_{k, m}^{N}+ \\
& +\sum_{i=3}^{\left\lfloor\frac{N+1}{2}\right\rfloor} \sum_{m=i}^{N-i} \sum_{j=i}^{N-m} \sum_{k=2}^{i-1} g_{i, j+m} \ell_{i} \ell_{k} c_{i, j}^{N} c_{k, m}^{N}
\end{aligned}
$$

and, finally, by changing, in the first multiple sum, the order of summation $\sum_{m} \sum_{i} \sum_{j} \sum_{k}$ to $\sum_{k} \sum_{m} \sum_{i} \sum_{j}$ and relabeling $(k, m, i, j) \mapsto(i, j, k, m)$ afterwards, one can finally write (19) in the following form

$$
\begin{aligned}
& \sum_{i=2}^{\left\lfloor\frac{N-1}{2}\right\rfloor} \sum_{j=i}^{\left\lfloor\frac{N-1}{2}\right\rfloor} \sum_{k=j+1}^{N-j} \sum_{m=k}^{N-j} g_{k, j+m} \ell_{i} \ell_{k} c_{i, j}^{N} c_{k, m}^{N}+ \\
& +\sum_{i=3}^{\left\lfloor\frac{N+1}{2}\right\rfloor} \sum_{m=i}^{N-i} \sum_{j=i}^{N-m} \sum_{k=2}^{i-1} g_{i, j+m} \ell_{i} \ell_{k} c_{i, j}^{N} c_{k, m}^{N}
\end{aligned}
$$

For the contribution (20) to $Q_{g}^{N}$ we have

$$
\begin{aligned}
- & \sum_{i=2}^{\left\lfloor\frac{N}{2}\right\rfloor} \sum_{j=i}^{N-i} \sum_{m=i}^{N-j} g_{i, j} \ell_{i}^{2} c_{i, j}^{N} c_{i, m}^{N}= \\
= & -\sum_{i=2}^{\left\lfloor\frac{N}{2}\right\rfloor} \sum_{j=i}^{\left\lfloor\frac{N}{2}\right\rfloor} \sum_{m=j}^{N-j} g_{i, j} \ell_{i}^{2} c_{i, j}^{N} c_{i, m}^{N}-\sum_{i=2}^{\left\lfloor\frac{N}{2}\right\rfloor} \sum_{m=i}^{\left\lfloor\frac{N}{2}\right\rfloor} \sum_{j=m}^{N-m} g_{i, j} \ell_{i}^{2} c_{i, j}^{N} c_{i, m}^{N} \\
& +\sum_{i=2}^{\left\lfloor\frac{N}{2}\right\rfloor} \sum_{j=i}^{\left\lfloor\frac{N}{2}\right\rfloor} g_{i, j} \ell_{i}^{2}\left(c_{i, j}^{N}\right)^{2}
\end{aligned}
$$

where the sums in the right-hand side correspond to the $(j, m)$-regions $S_{1}, S_{2}$, and $S_{3}$ in Figure 3, respectively. 


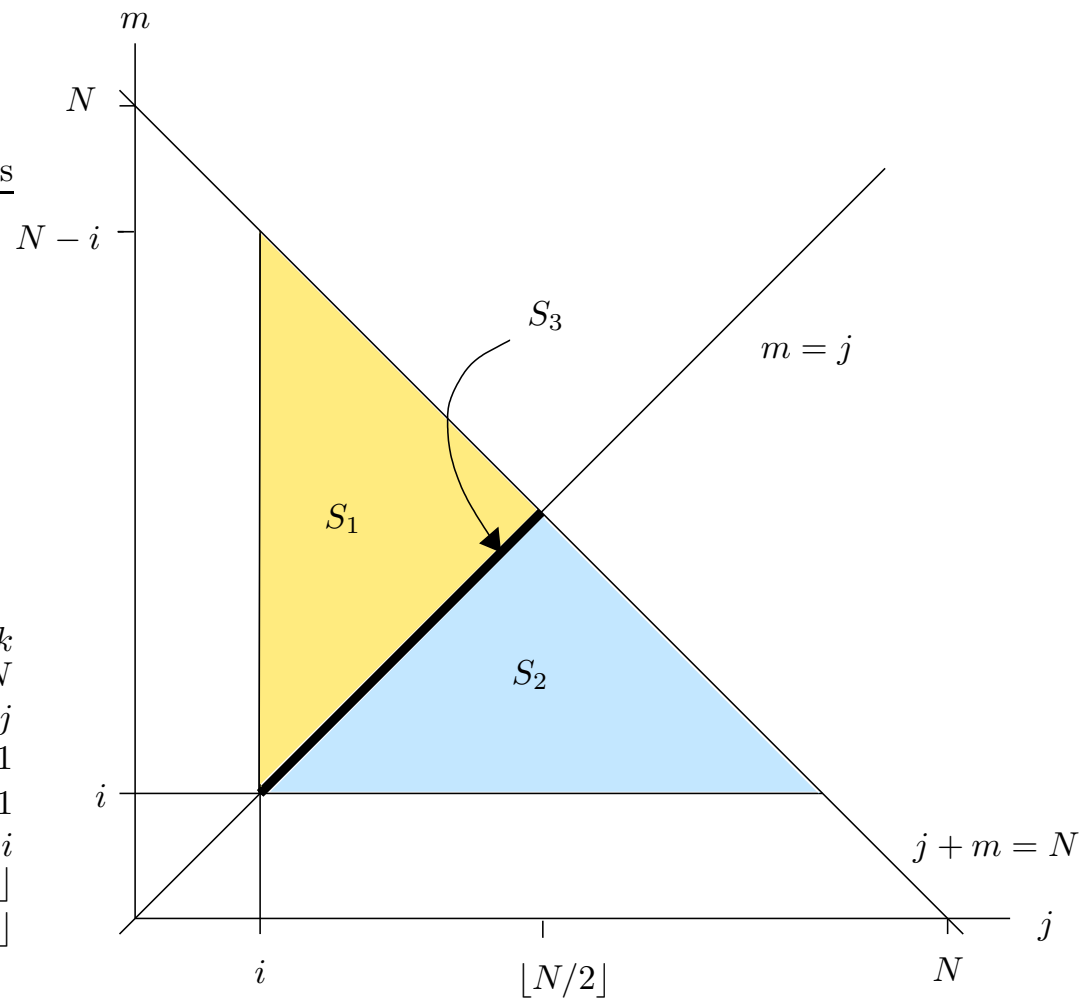

Figure 3: Regions of the $(j, m)$-space used in the decomposition of sum (26) of $Q_{g}^{N}$. (Note that $S_{1} \cap S_{2}=S_{3}$.)

By changing notation $(m, j) \mapsto(j, m)$ in the second multiple sum in (26) we conclude that (20) is equal to

$$
-\sum_{i=2}^{\left\lfloor\frac{N}{2}\right\rfloor} \sum_{j=i}^{\left\lfloor\frac{N}{2}\right\rfloor} \sum_{m=j}^{N-j}\left(g_{i, j}+g_{i, m}\right) \ell_{i}^{2} c_{i, j}^{N} c_{i, m}^{N}+\sum_{i=2}^{\left\lfloor\frac{N}{2}\right\rfloor} \sum_{j=i}^{\left\lfloor\frac{N}{2}\right\rfloor} g_{i, j} \ell_{i}^{2}\left(c_{i, j}^{N}\right)^{2}
$$

Observe that (21) is canceled by the last multiple sum in (27).

Finally, for the last sum in $Q_{g}^{N}$, namely (22), we have, by considering the triangular pyramid defined by

$$
\{(i, j, k): 2 \leq i \leq N-3, i \leq j \leq N-3,2 \leq k \leq N-1-j\}
$$


and separating the parts with $k<i$ and with $k>i$,

$$
\begin{aligned}
-\sum_{i=2}^{N-2} \sum_{j=i}^{N-2} \sum_{\substack{k=2 \\
k \neq i}}^{N-j} \sum_{m=k}^{N-j} g_{i, j} \ell_{i} \ell_{k} c_{i, j}^{N} c_{k, m}^{N}= \\
=-\sum_{k=2}^{\left\lfloor\frac{N-1}{2}\right\rfloor} \sum_{i=k+1}^{N-k} \sum_{j=i}^{N-k} \sum_{m=k}^{N-j} g_{i, j} \ell_{i} \ell_{k} c_{i, j}^{N} c_{k, m}^{N}+ \\
-\sum_{i=2}^{\left\lfloor\frac{N-1}{2}\right\rfloor} \sum_{k=i+1}^{N-i} \sum_{j=i}^{N-k} \sum_{m=k}^{N-j} g_{i, j} \ell_{i} \ell_{k} c_{i, j}^{N} c_{k, m}^{N}
\end{aligned}
$$

which, by changing the notation in the first multiple sum of the right-hand side $(k, m, i, j) \mapsto(i, j, k, m)$ and subsequently changing the order of summation $\sum_{m} \sum_{j}$ to $\sum_{j} \sum_{m}$, can be written as

$$
-\sum_{i=2}^{\left\lfloor\frac{N-1}{2}\right\rfloor} \sum_{k=i+1}^{N-i} \sum_{j=i}^{N-k} \sum_{m=k}^{N-j}\left(g_{i, j}+g_{k, m}\right) \ell_{i} \ell_{k} c_{i, j}^{N} c_{k, m}^{N}
$$

Using (24), (25), (27) and (28) in (18)-(22) we can write

$$
\begin{aligned}
Q_{p, q}^{N}\left(c^{N}\right)= & \sum_{i=2}^{\left\lfloor\frac{N}{2}\right\rfloor} \sum_{j=i}^{\left\lfloor\frac{N}{2}\right\rfloor} \sum_{m=j}^{N-j} g_{i+1, j+m} \ell_{i}^{2} c_{i, j}^{N} c_{i, m}^{N} \\
& +\sum_{i=2}^{\left\lfloor\frac{N-1}{2}\right\rfloor} \sum_{j=i}^{\left\lfloor\frac{N-1}{2}\right\rfloor} \sum_{k=j+1}^{N-j} \sum_{m=k}^{N-j} g_{k, j+m} \ell_{i} \ell_{k} c_{i, j}^{N} c_{k, m}^{N} \\
& +\sum_{i=3}^{\left\lfloor\frac{N+1}{2}\right\rfloor} \sum_{m=i}^{N-i} \sum_{j=i}^{N-m} \sum_{k=2}^{i-1} g_{i, j+m} \ell_{i} \ell_{k} c_{i, j}^{N} c_{k, m}^{N} \\
& -\sum_{i=2}^{\left\lfloor\frac{N}{2}\right\rfloor} \sum_{j=i}^{\left\lfloor\frac{N}{2}\right\rfloor} \sum_{m=j}^{N-j}\left(g_{i, j}+g_{i, m}\right) \ell_{i}^{2} c_{i, j}^{N} c_{i, m}^{N} \\
& -\sum_{i=2}^{\left\lfloor\frac{N-1}{2}\right\rfloor} \sum_{k=i+1}^{N-i} \sum_{j=i}^{N-k} \sum_{m=k}^{N-j}\left(g_{i, j}+g_{k, m}\right) \ell_{i} \ell_{k} c_{i, j}^{N} c_{k, m}^{N}
\end{aligned}
$$

From this it imediatly follows that the sum of (29) with (32) results in (13). To conclude the proof we are left to establish that adding (30), (31), and (33) must result in (14). To this end we start by considering (31) and sucessively perform the following transformations: change the order of summation $\sum_{m} \sum_{j} \mapsto \sum_{j} \sum_{m}$, changing the notation $(i, j, k, m) \mapsto(k, m, i, j)$, and changing again $\sum_{k} \sum_{i} \mapsto \sum_{i} \sum_{k}$ and $\sum_{m} \sum_{j} \mapsto \sum_{j} \sum_{m}$. We then obtain the 
following alternative expression for (31),

$$
\sum_{i=2}^{\left\lfloor\frac{N-1}{2}\right\rfloor} \sum_{k=i+1}^{\left\lfloor\frac{N+1}{2}\right\rfloor} \sum_{j=k}^{N-k} \sum_{m=k}^{N-j} g_{k, j+m} \ell_{i} \ell_{k} c_{i, j}^{N} c_{k, m}^{N}
$$

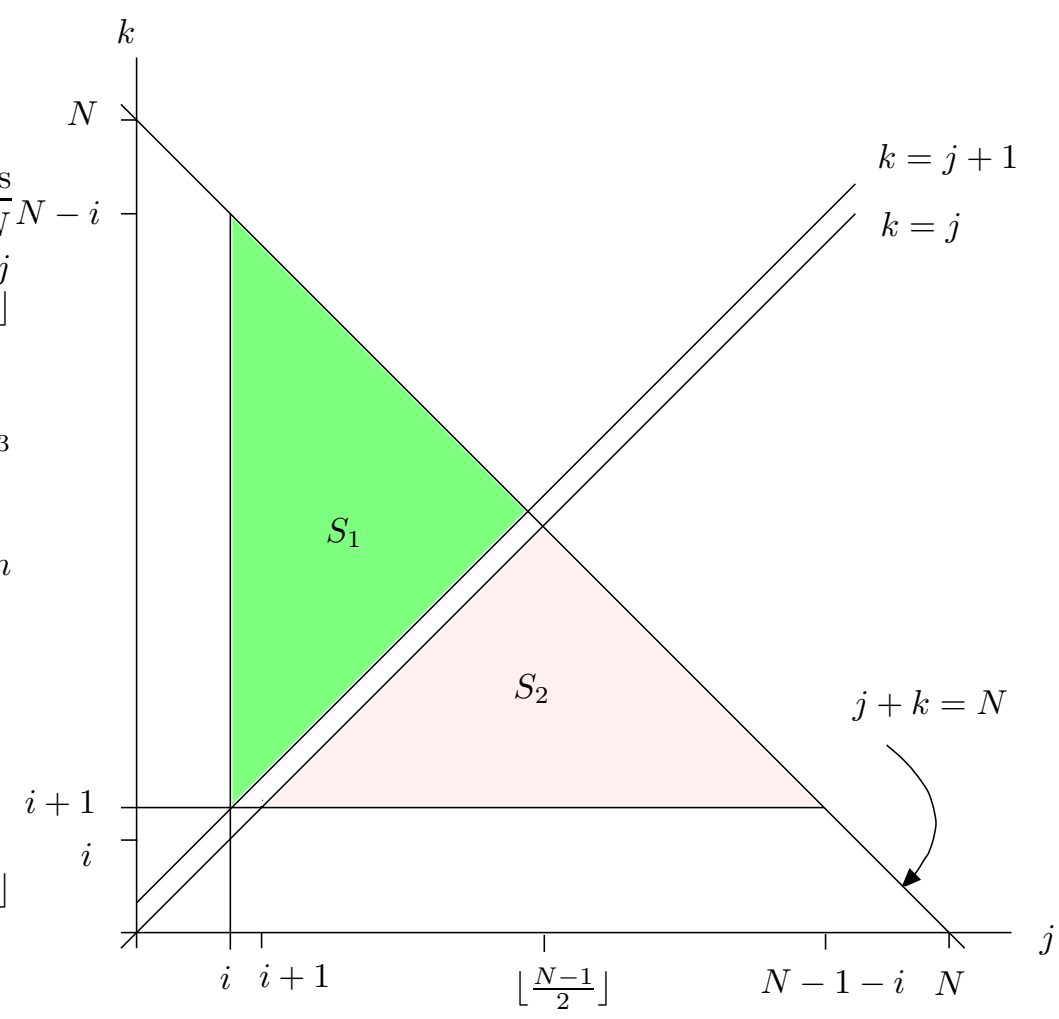

Figure 4: Regions correspondent to the sums (30) and (34).

Ploting the $(j, k)$-regions of the sums in (30) and (34) in Figure 4 we imediatly recognize that those two terms can be combined into

$$
\sum_{i=2}^{\left\lfloor\frac{N-1}{2}\right\rfloor} \sum_{k=i+1}^{N-i} \sum_{j=i}^{N-k} \sum_{m=k}^{N-j} g_{k, j+m} \ell_{i} \ell_{k} c_{i, j}^{N} c_{k, m}^{N}
$$

which, together with (33), gives (14) and concludes the proof. 
Corollary 1 With the conditions of Proposition 3 we have

$$
\begin{array}{llrl}
\frac{d}{d t}\left\|c^{N}(t)\right\|_{0,1} & =\dot{\rho}^{N}\left(c^{N}(t)\right)=0 & & \text { (conservation of mass) } \\
\frac{d}{d t}\left\|c^{N}(t)\right\|_{0,0} & \leq 0 . & & \text { (decrease of the total number of clusters) } \\
\frac{d}{d t}\left\|c^{N}(t)\right\|_{1,0} & \leq 0 . &
\end{array}
$$

Proof: Just take $g_{i, j}=j$ for the first case, $g_{i, j}=1$ for the second, and $g_{i, j}=i$ for the last, in the expression in the statement of Proposition 3.

We can now use the conservation of mass to get the global existence of solutions to Cauchy problems for (8)-(9).

Proposition 4 Let $c^{N}=\left(c_{i, j}^{N}\right)$ be the solution to (8)-(9) with nonnegative initial condition $c_{0}^{N}$. Then, the maximal forward interval of definition of $c^{N}$ is $[0, \infty)$

Proof: Let $0<T<\infty$ be such that $[0, T)$ is the maximal forward interval of $c^{N}$. Then, by Corollary 1 , we know that, for all $(i, j) \in \Xi_{N}$ and $t \in[0, T)$,

$$
c_{i, j}^{N}(t) \leq \frac{1}{j} \rho^{N}\left(c_{0}^{N}\right)=\frac{1}{j}\left\|c_{0}^{N}\right\|_{0,1} \leq\left\|c_{0}\right\|_{0,1},
$$

and, since the right hand side of (8)-(9) is a polynomial function of the components $c_{i, j}^{N}$ of $c^{N}$, we also conclude that $\dot{c}_{i, j}^{N}(t)$ is also bounded. Hence, the solution can be extended to $\left[0, T^{*}\right)$ for some $T^{*}>T$, contradicting the maximality of $[0, T)$. This implies that $T=\infty$ and concludes the proof.

\section{$3 \quad$ Existence and Uniqueness}

The proof of existence of solutions to (2)-(5) follows the same basic ideas that were used in [20] or in [19]: first we get the existence of continuous functions $c_{i, j}$ that are limits of $c_{i, j}^{N_{\nu}}$ when $N_{\nu} \rightarrow \infty$, for some integer sequence $\left(N_{\nu}\right)$; Then we prove the limit function $c=\left(c_{i, j}\right)$ thus obtained is a solution to the integral version of (2)-(5).

Proposition 5 Let $0 \leq \ell_{j} \leq A i^{\alpha}$, for some $\alpha \in[0,1)$ and $A \geq 0$. Let $c_{0} \in$ $\left(X_{0,1} \cap X_{1,0}\right)^{+}$. Then there exists a solution $c$ of (2)-(5) satisfying $c(0)=c_{0}$.

Proof: For each $N \in \mathbb{Z}^{+}$let $c_{0}^{N}=\left(c_{0 i, j} \delta_{(i, j) \in \Xi_{N}}\right)$ and denote by $c^{N}=\left(c_{i, j}^{N}\right)$ the global solution of (8)-(9) with initial condition equal to $c_{0}^{N}$. Consider $c^{N}$ as an element of $X_{p, q}$ by defining as zero all $c_{i, j}^{N}$ with $(i, j) \notin \Xi_{N}$. Using Corollary 1 a version of (35) for the norm $\|\cdot\|_{1,0}$ is easily obtained:

$$
c_{i, j}^{N}(t) \leq \frac{1}{i}\left\|c^{N}(t)\right\|_{1,0} \leq\left\|c_{0}^{N}\right\|_{1,0} \leq\left\|c_{0}\right\|_{1,0},
$$


By (8) and (36),

$$
\begin{aligned}
\left|\dot{c}_{1,1}^{N}\right| & \leq 2 \ell_{1}^{2}\left|c_{1,1}^{N}\right|+\ell_{1}\left|c_{1,1}^{N}\right| \sum_{i=2}^{N-1} \sum_{j=i}^{N-1} \ell_{i}\left|c_{i, j}^{N}\right| \\
& \leq 2 A^{2}\left|c_{1,1}^{N}\right|\left(\left|c_{1,1}^{N}\right|+\sum_{i=2}^{N} \sum_{j=i}^{N} i\left|c_{i, j}^{N}\right|\right) \\
& \leq 2 A^{2}\left\|c_{0}^{N}\right\|_{1,0}^{2} \leq 2 A^{2}\left\|c_{0}\right\|_{1,0}^{2}
\end{aligned}
$$

and similarly, applying (36) to (9),

$$
\left|\dot{c}_{i, j}^{N}\right| \leq 8 A^{2}\left\|c_{0}\right\|_{1,0}^{2}
$$

From these inequalities it follows that, for all $(i, j) \in \Xi$ and all $t \in[0, \tau]$ the derivatives $\dot{c}_{i, j}^{N}(t)$ are bounded by a constant depending only on $A, \theta$, and $c_{0}$. By Lagrange's theorem this implies equicontinuity of $\left(c_{i, j}^{N}\right)$ in $[0, \tau]$. Furthermore, either (35) or (36) imply equiboundedness. By the Ascoli-Arzela theorem we conclude that, for each $(i, j) \in \Xi$, there exists a continuous function $c_{i, j}$ and an integer sequence $N_{\mu} \rightarrow \infty$ such that $c_{i, j}^{N_{\mu}} \rightarrow c_{i, j}$ in $C([0, \theta])$. Starting with a fixed $(i, j) \in \Xi$, for instance $(1,1)$, and proceeding by an appropriate diagonal argument we can guarantee the existence of a sequence $N_{\nu} \rightarrow \infty$ such that, $c_{i, j}^{N_{\nu}} \rightarrow c_{i, j}$, in $C([0, \tau])$, for all $(i, j) \in \Xi$.

The function $c=\left(c_{i, j}\right)$ defined by the limit process just described is the natural candidate for a solution to the Cauchy problem for (2)-(5) with initial condition $c_{0}$. Let $t \in[0, \tau)$ be arbitrary. Integrating (8)-(9) between 0 and $t$ we should be able to pass to the limit $N=N_{\nu} \rightarrow \infty$ and obtain the integral version of (2)-(5) as the equation satisfied by the limit function $c$. This was the approach employed in several works on cluster equations (eg, [16, 19, 17, 21]) and can also be used here. In what follows we just describe outline its applycation to the present case. Since the details are very similar to what was done in the references cited we shall not present the details.

There are two different kind of terms in the integrated version of (8)-(9) that require separated treatment: the simpler ones, like

$$
-\ell_{1}^{2} \int_{0}^{t}\left(c_{1,1}^{N_{\nu}}\right)^{2}
$$

in the equation for $c_{1,1}^{N_{\nu}}$, and all but the fifth and the eighth terms in the right hand side of the integrated version of (9), can be easily proven to converge to the corresponding terms in (2)-(5), such as

$$
-\ell_{1}^{2} \int_{0}^{t}\left(c_{1,1}\right)^{2}
$$

for the case above; this is done by using the bound (35) or (36), the pointwise convergence of $c_{i, j}^{N_{\nu}}$ to $c_{i, j}$, and the dominated convergence theorem. A less 
simpler case is that of the term

$$
-\ell_{1} c_{1,1}^{N_{\nu}} \sum_{i=2}^{N_{\nu}-1} \sum_{j=i}^{N_{\nu}-1} \ell_{i} c_{i, j}^{N_{\nu}}
$$

in the equation for $c_{1,1}^{N_{\nu}}$, as well as the fifth and eighth terms in the right hand side of the equation for $c_{i, j}^{N_{\nu}}$. For these terms the kind of estimates used in [17, pp 905-906] or in [21, pp 3403] can be readly applied to get the result.

As in the study of existence of solutions, the basic tool for the uniqueness was developed in [16]: With the conditions of Proposition 5, assume $c$ and $d$ are two solutions to the initial value problem for (2)-(5) with initial datum $c^{0}$, let $x=c-d$ and define the quantity

$$
\theta_{n}:=\sum_{(i, j) \in \Xi} i^{\beta}\left|x_{i, j}\right|,
$$

where $\beta=1-\alpha$. Using the same type of algebraic manipulations presented in Section 2 one can write

$$
\begin{aligned}
\theta_{n}(t)= & \int_{0}^{t}\left(g_{2,2}-2 g_{1,1}\right) \ell_{1}^{2}\left(c_{1,1}+d_{1,1}\right) x_{i, j} \\
= & \int_{0}^{t} \sum_{i=2}^{n-1} \sum_{j=i}^{n-1}\left(g_{i, j+1}-g_{i, j}-g_{1,1}\right) \ell_{1} \ell_{i}\left(x_{1,1} c_{i, j}+d_{1,1} x_{i, j}\right) \\
& +\int_{0}^{t} \sum_{i=2}^{\left\lfloor\frac{n}{2}\right\rfloor} \sum_{j=i}^{\left\lfloor\frac{n}{2}\right\rfloor} \sum_{m=j}^{n-j}\left(g_{i+1, j+m}-g_{i, j}-g_{i, m}\right) \ell_{i}^{2}\left(c_{i, j} x_{i, m}+x_{i, j} d_{i, m}\right) \\
& +\int_{0}^{t} \sum_{i=2}^{\left\lfloor\frac{n-1}{2}\right\rfloor} \sum_{k=i+1}^{n-i} \sum_{j=i}^{n-k} \sum_{m=k}^{n-j}\left(g_{k, j+m}-g_{i, j}-g_{k, m}\right) \ell_{i} \ell_{k}\left(c_{i, j} x_{k, m}+x_{i, j} d_{k, m}\right) \\
& +\int_{0}^{t} S_{n}(c, d)
\end{aligned}
$$


where $g_{i, j}=i^{\beta} \operatorname{sgn}\left(x_{i, j}\right)$, and

$$
\begin{aligned}
S_{n}(c, d)= & -\sum_{i=2}^{n} g_{i, n+1} \ell_{1} \ell_{i}\left(c_{1,1} c_{i, n+1}-d_{1,1} d_{i, n+1}\right) \\
& -\sum_{j=n+1}^{\infty} \sum_{i=2}^{j} g_{1,1} \ell_{1} \ell_{i}\left(c_{1,1} c_{i, j}+d_{1,1} d_{i, j}\right) \\
& -\sum_{j=\left\lfloor\frac{n}{2}\right\rfloor+1}^{n} \sum_{i=2}^{j} g_{i, j} \ell_{i}^{2}\left(c_{i, j}^{2}-d_{i, j}^{2}\right) \\
& -\sum_{\Omega_{1, n}} g_{i, j} \ell_{i}^{2}\left(c_{i, j} c_{i, m}-d_{i, j} d_{i, m}\right) \\
& -\sum_{\Omega_{2, n}} g_{i, j} \ell_{i} \ell_{k}\left(c_{i, j} c_{k, m}-d_{i, j} d_{k, m}\right)
\end{aligned}
$$

with

$$
\Omega_{1, n}=\{(i, j, m): 2 \leq i \leq j \leq n, i \vee j \leq m<\infty\}
$$

and

$$
\begin{aligned}
\Omega_{2, n}= & \{(i, j, k, m): 2 \leq i \leq j \leq n, 2 \leq k \leq m<\infty, k \neq i\} \backslash \\
& \backslash\{(i, j, k, m): 2 \leq i \leq j \leq n-2,2 \leq k \leq m \leq n-j, k \neq i\} .
\end{aligned}
$$

If we impose the restriction $\alpha \in[0,1 / 2]$ we have, after some further manipulations, but without new essential difficulties, $\int_{0}^{t} S_{n}(c, d) \rightarrow 0$ as $n \rightarrow \infty$, and the remaining terms in the right-hand side of the equation for $\theta_{n}$ can be bounded above by $12 A^{2}\left\|c^{0}\right\|_{1,0} \theta_{n}$. Hence, denoting by $\theta$ the limit of $\theta_{n}$ as $n \rightarrow \infty$, we obtain, after taking the limit $n \rightarrow \infty$,

$$
\theta(t) \leq 12 A^{2}\left\|c^{0}\right\|_{1,0} \int_{0}^{t} \theta(s) d s,
$$

which, by Gronwall's inequality, concludes the proof of the following uniqueness result.

Proposition 6 Let $0 \leq \ell_{j} \leq A i^{\alpha}$, for some $\alpha \in[0,1 / 2]$ and $A \geq 0$. Then, for each initial condition $c_{0} \in\left(X_{0,1} \cap X_{1,0}\right)^{+}$, there is only one solution $c$ of (2)-(5) satisfying $c(0)=c_{0}$.

\section{Convergence to Equilibria}

We first study the long time approach to equilibria of each component of the solution function $c=\left(c_{i, j}\right)$. 
Proposition 7 Let $\ell_{j}>0$ for all $j$. Let $c=\left(c_{i, j}\right)$ be any solution to (2)-(5) with nonnegative initial data. Then $c_{i, j}(t) \longrightarrow 0$ as $t \rightarrow \infty$, for all $(i, j) \in \Xi$.

Proof: The main idea of the proof is the joint use of the time monotonicity of the quantities $P_{N}(c(\cdot))$ and $R_{N}(c(\cdot))$, where $P_{1}(c)=R_{1}(c)=c_{1,1}$, and, for $N \geq 2$,

$$
P_{N}(c):=c_{1,1}+\sum_{i=2}^{N} \sum_{j=i}^{N} j c_{i, j} \quad \text { and } \quad R_{N}(c):=c_{1,1}+\sum_{i=2}^{N} \sum_{j=i}^{N} c_{i, j} .
$$

The case $N=1$ is trivial, since, by (2), the function $c_{1,1}(t)$ is monotonically decreasing.

Let us now turn to the case $N \geq 2$. We start with the study of $R_{N}$. For all $t, \tau>0$, we can write, after some algebraic manipulations similar to those performed to obtain (15)-(22),

$$
\begin{aligned}
& R_{N}(c(t+\tau))-R_{N}(c(t))= \\
& =-\int_{t}^{t+\tau} \ell_{1}^{2} c_{1,1}^{2}+ \\
& +\int_{t}^{t+\tau} \ell_{1} c_{1,1}\left(-\sum_{i=2}^{\infty} \sum_{j=i}^{\infty} \ell_{i} c_{i, j}+\sum_{i=2}^{N-1} \sum_{j=i+1}^{N} \ell_{i} c_{i, j-1}-\sum_{i=2}^{N} \sum_{j=i}^{N} \ell_{i} c_{i, j}\right)+ \\
& +\int_{t}^{t+\tau}\left(\sum_{i=3}^{\left\lfloor\frac{N}{2}\right\rfloor+1} \sum_{j=2 i-2}^{N} \sum_{m=\left\lfloor\frac{j+1}{2}\right\rfloor}^{j-i+1} \ell_{i-1}^{2} c_{i-1, m} c_{i-1, j-m}+\right. \\
& +\sum_{i=3}^{N-2} \sum_{j=i+2}^{N} \sum_{m=i}^{j-2} \sum_{k=2}^{(i-1) \wedge(j-m)} \ell_{i} \ell_{k} c_{i, m} c_{k, j-m}- \\
& \quad-\sum_{i=2}^{N} \sum_{j=i}^{N} \sum_{m=i}^{\infty} \ell_{i}^{2} c_{i, j} c_{i, m}-\sum_{i=2}^{N} \sum_{j=i}^{N} \ell_{i}^{2} c_{i, j}^{2}- \\
& \left.\quad-\sum_{i=2}^{N} \sum_{j=i}^{N} \sum_{\substack{k=2 \\
k \neq i}}^{\infty} \sum_{m=k}^{\infty} \ell_{i} \ell_{k} c_{i, j} c_{k, m}\right)
\end{aligned}
$$

Comparing the right-hand side of (37) with (15)-(17) with $p=q=0$ we conclude that the right hand side of (37) is nonpositive because all the positive terms are the same (with $c_{i, j}^{N}$ changed to $c_{i, j}$ ) and there are a lot more negative terms contributing to the right hand side of (37). Thus, the right hand side of (37) is not larger than the terms (15)-(17) computed with $c_{i, j}$ in the place of $c_{i, j}^{N}$ and $p=q=0$. Since, by Corolary 1 , the result of these computations is nonpositive, we conclude that $R_{N}(c(t+\tau)) \leq R_{N}(c(t))$ and hence $R_{N}(\cdot)$ is monotonically decreasing along solutions. 
We now consider the behaviour of $P_{N}$. In a way similar to that used in the study of $R_{N}$ we obtain, after some algebraic manipulations

$$
\begin{gathered}
P_{N}(c(t+\tau))-P_{N}(c(t))= \\
=-\int_{t}^{t+\tau} \ell_{1} c_{1,1}\left(\sum_{j=N}^{\infty} \sum_{i=2}^{j} \ell_{i} c_{i, j}+N \sum_{i=2}^{N} \ell_{i} c_{i, N}\right)+ \\
+\int_{t}^{t+\tau}\left(\sum_{i=3}^{\left\lfloor\frac{N}{2}\right\rfloor+1} \sum_{j=2 i-2}^{N} \sum_{m=\left\lfloor\frac{j+1}{2}\right\rfloor}^{j-i+1} j \ell_{i-1}^{2} c_{i-1, m} c_{i-1, j-m}+\right. \\
+\sum_{i=3}^{N-2} \sum_{j=i+2}^{N} \sum_{m=i}^{j-2} \sum_{k=2}^{(i-1) \wedge(j-m)} j \ell_{i} \ell_{k} c_{i, m} c_{k, j-m}- \\
-\sum_{i=2}^{N} \sum_{j=i}^{N} \sum_{\substack{m=i \\
m}}^{\infty} j \ell_{i}^{2} c_{i, j} c_{i, m}-\sum_{i=2}^{N} \sum_{j=i}^{N} j \ell_{i}^{2} c_{i, j}^{2}- \\
\left.-\sum_{i=2}^{N} \sum_{j=i}^{N} \sum_{\substack{k=2 \\
k \neq i}}^{\infty} \sum_{m=k}^{\infty} j \ell_{i} \ell_{k} c_{i, j} c_{k, m}\right)
\end{gathered}
$$

As was done in the case of $R_{N}$, we can now easily compare the terms in the last integral in (38) with $Q_{g}^{N}(c)$ with $g_{i, j}=j$ and conclude that the present sum is not greater than $Q_{j}^{N}(c)$. Since, by the proof of Proposition 3 , we know that $Q_{j}^{N}(c) \leq 0$, we can conclude that $P_{N}(\cdot)$ is decreasing along solutions.

Both $P_{N}(\cdot)$ and $R_{N}(\cdot)$ are nonnegative when evaluated in nonnegative solutions. Hence, they are both decreasing and bounded below, which imply they converge to some nonnegative real numbers: $P_{N}(c(t)) \rightarrow \alpha_{N}$ and $R_{N}(c(t)) \rightarrow \beta_{N}$. Note that, for all $N, \alpha_{N} \geq \beta_{N}$. We want to prove that $\alpha_{N}=0$ and $\beta_{N}=0$, for all $N \geq 1$, and so $c_{i, j}(t) \rightarrow 0$ as $t \rightarrow \infty$, for all $(i, j) \in \Xi$.

For $N=1$, where we naturally have $\alpha_{1}=\beta_{1}$ we note that, since $c_{1,1}(t)$ is decreasing, $c_{1,1}(t) \geq \alpha_{1}$. Assume $\alpha_{1}>0$. Then,

$$
\begin{aligned}
c_{1,1}(t+\tau)-c_{1,1}(t) & =-\int_{t}^{t+\tau}\left(2 \ell_{1}^{2} c_{1,1}^{2}+\ell_{1} c_{1,1} \sum_{i=2}^{\infty} \sum_{j=i}^{\infty} \ell_{i} c_{i, j}\right) \\
& \leq-2 \ell_{1}^{2} \int_{t}^{t+\tau} c_{1,1}^{2} \leq-2 \ell_{1}^{2} \alpha_{1}^{2} \tau<0 .
\end{aligned}
$$

By letting $t \rightarrow \infty$ we conclude that, for all $\tau>0$,

$$
0 \leq-2 \ell_{1} \alpha_{1}^{2} \tau<0
$$

This contradiction implies that we must have $\alpha_{1}=0$. 
For $N \geq 2$ we use the expression for $P_{N}$ given in (38) and will relate it to $R_{N}$. We start by observing that

$$
\begin{aligned}
& \sum_{i=2}^{N} \sum_{j=i}^{N} \sum_{m=i}^{\infty} j \ell_{i}^{2} c_{i, j} c_{i, m}+\sum_{i=2}^{N} \sum_{j=i}^{N} j \ell_{i}^{2} c_{i, j}^{2}+\sum_{i=2}^{N} \sum_{j=i}^{N} \sum_{\substack{k=2 \\
k \neq i}}^{\infty} \sum_{m=k}^{\infty} j \ell_{i} \ell_{k} c_{i, j} c_{k, m} \geq \\
& \geq \sum_{i=2}^{N} \sum_{j=i}^{N} j \ell_{i}^{2} c_{i, j} \sum_{m=i}^{N} c_{i, m}+\sum_{i=2}^{N} \sum_{j=i}^{N} j \ell_{i} c_{i, j} \sum_{\substack{k=2 \\
k \neq i}}^{N} \sum_{m=k}^{N} \ell_{k} c_{k, m} \\
& \geq \min _{2 \leq i \leq N}\left\{\ell_{i}^{2}\right\} \sum_{i=2}^{N} \sum_{j=i}^{N} j c_{i, j} \sum_{m=i}^{N} c_{i, m}+ \\
& \quad+\min _{2 \leq i \leq N}\left\{\ell_{i}^{2}\right\} \sum_{i=2}^{N} \sum_{j=i}^{N} j c_{i, j} \sum_{\substack{k=2 \\
k \neq i}}^{N} \sum_{m=k}^{N} c_{k, m} \\
&= \min _{2 \leq i \leq N}\left\{\ell_{i}^{2}\right\}\left(\sum_{i=2}^{N} \sum_{j=i}^{N} j c_{i, j}\right)\left(\sum_{k=2}^{N} \sum_{k=m}^{N} c_{k, m}\right) \\
& \geq \min _{2 \leq i \leq N}\left\{\ell_{i}^{2}\right\}\left(\sum_{i=2}^{N} \sum_{j=i}^{N} c_{i, j}\right)^{2}
\end{aligned}
$$

We also note, by the proof of Proposition 3 , that $Q_{j}^{N}(c)=0$, and so we can write

$$
\begin{aligned}
\sum_{i=3}^{\left\lfloor\frac{N}{2}\right\rfloor+1} \sum_{j=2 i-2}^{N} \sum_{m=\left\lfloor\frac{j+1}{2}\right\rfloor}^{j-i+1} j \ell_{i-1}^{2} c_{i-1, m} c_{i-1, j-m}+ & \\
+\sum_{i=3}^{N-2} \sum_{j=i+2}^{N} \sum_{m=i}^{j-2} \sum_{k=2}^{(i-1) \wedge(j-m)} j \ell_{i} \ell_{k} c_{i, m} c_{k, j-m}= & \sum_{i=2}^{\left\lfloor\frac{N}{2}\right\rfloor} \sum_{j=i}^{N-i} \sum_{m=i}^{N-j} j \ell_{i}^{2} c_{i, j} c_{i, m} \\
& +\sum_{i=2}^{\left\lfloor\frac{N}{2}\right\rfloor} \sum_{j=i}^{\left\lfloor\frac{N}{2}\right\rfloor} j \ell_{i}^{2} c_{i, j}^{2} \\
& +\sum_{i=2}^{N-2} \sum_{j=i}^{N-2} \sum_{\substack{k=2 \\
k \neq i}}^{N-j} \sum_{m=k}^{N-j} j \ell_{i} \ell_{k} c_{i, j} c_{k, m}
\end{aligned}
$$


(again with the convention that a sum is equal to zero if its upper index is smaller than its lower.) These observations can be used to write (38) as follows

$$
\begin{aligned}
& P_{N}(c(t+\tau))-P_{N}(c(t))= \\
& =-\int_{t}^{t+\tau} \ell_{1} c_{1,1}\left(\sum_{j=N}^{\infty} \sum_{i=2}^{j} \ell_{i} c_{i, j}+N \sum_{i=2}^{N} \ell_{i} c_{i, N}\right) \\
& -\int_{t}^{t+\tau}\left(\sum_{i=2}^{N} \sum_{j=i}^{N} \sum_{m=i}^{\infty} j \ell_{i}^{2} c_{i, j} c_{i, m}+\sum_{i=2}^{N} \sum_{j=i}^{N} j \ell_{i}^{2} c_{i, j}^{2}+\right. \\
& +\sum_{i=2}^{N} \sum_{j=i}^{N} \sum_{\substack{k=2 \\
k \neq i}}^{\infty} \sum_{m=k}^{\infty} j \ell_{i} \ell_{k} c_{i, j} c_{k, m}- \\
& -\sum_{i=2}^{\left\lfloor\frac{N}{2}\right\rfloor} \sum_{j=i}^{N-i} \sum_{m=i}^{N-j} j \ell_{i}^{2} c_{i, j} c_{i, m}-\sum_{i=2}^{\left\lfloor\frac{N}{2}\right\rfloor} \sum_{j=i}^{\left\lfloor\frac{N}{2}\right\rfloor} j \ell_{i}^{2} c_{i, j}^{2}- \\
& \left.-\sum_{i=2}^{N-2} \sum_{j=i}^{N-2} \sum_{\substack{k=2 \\
k \neq i}}^{N-j} \sum_{m=k}^{N-j} j \ell_{i} \ell_{k} c_{i, j} c_{k, m}\right) \\
& \leq \int_{t}^{t+\tau}\left(\sum_{i=2}^{\left\lfloor\frac{N}{2}\right\rfloor} \sum_{j=i}^{N-i} \sum_{m=i}^{N-j} j \ell_{i}^{2} c_{i, j} c_{i, m}+\sum_{i=2}^{\left\lfloor\frac{N}{2}\right\rfloor} \sum_{j=i}^{\left\lfloor\frac{N}{2}\right\rfloor} j \ell_{i}^{2} c_{i, j}^{2}+\right. \\
& \left.+\sum_{i=2}^{N-2} \sum_{j=i}^{N-2} \sum_{\substack{k=2 \\
k \neq i}}^{N-j} \sum_{m=k}^{N-j} j \ell_{i} \ell_{k} c_{i, j} c_{k, m}\right)- \\
& -\min _{2 \leq i \leq N}\left\{\ell_{i}^{2}\right\} \int_{t}^{t+\tau}\left(\sum_{i=2}^{N} \sum_{j=i}^{N} c_{i, j}\right)^{2}
\end{aligned}
$$

Note that the integrand of (40) depends only on terms $c_{i, j}$ with $(i, j) \in \Xi_{N-1}$. We thus can use the induction principle to complete the proof. Suppose that for $(i, j) \in \Xi_{N-1}$ we have $c_{i, j}(t) \rightarrow 0$ as $t \rightarrow \infty$. Observing that

$$
\sum_{i=2}^{N} \sum_{j=i}^{N} c_{i, j}(t)=R_{N}(c(t))-c_{1,1}(t) \rightarrow \beta_{N} \quad \text { as } \quad t \rightarrow \infty,
$$

we have, after applying limits as $t \rightarrow \infty$ to (39)-(41),

$$
0 \leq-\min _{2 \leq i \leq N}\left\{\ell_{i}^{2}\right\} \lim _{t \rightarrow \infty} \int_{t}^{t+\tau}\left(R_{N}(c)-c_{1,1}\right)^{2} .
$$

If $\beta_{N}>0$ then, for all $t$ sufficiently large, $R_{N}(c(s))-c_{1,1}(s)>\frac{1}{2} \beta_{N}$ for all $s>t$. This implies that

$$
0=\lim _{t \rightarrow \infty} \int_{t}^{t+\tau}\left(R_{N}(c)-c_{1,1}\right)^{2}>\frac{1}{4} \beta_{N}^{2} \tau
$$


which is absurd since by hypothesis $\tau>0$. Thus we must have $\beta_{N}=0$ i.e., $c_{i, j}(t) \rightarrow 0$ as $t \rightarrow \infty$ for all $(i, j) \in \Xi_{N}$, and hence the result follows by induction.

We now turn our attention to the behaviour of sets of clusters with a fixed total order. Denoting by $N_{i}(c)$ the total number of clusters of order $i$, previously defined in (7), we prove the following

Proposition 8 Let $\ell_{j}>0$ for all $j$. Let $c=\left(c_{i, j}\right)$ be any solution to (2)-(5) with nonnegative initial data. Then, for the functional $N_{i}(c)$ defined above, we have $N_{i}(c(t)) \longrightarrow 0$ as $t \rightarrow \infty$, for all $i \geq 1$.

Proof: For all $p \geq 1$, define the functionals

$$
\mathcal{N}_{p}:=\sum_{i=1}^{p} N_{i}
$$

The main tool of the proof is to exploit the monotonicity of $\mathcal{N}_{p}$ that we establish first. For $p=1$, by (2), $\mathcal{N}_{1}=N_{1}=c_{1,1}$ is monotonically decreasing. To get the result for general $p \geq 2$ we need first to consider the truncated quantities

$$
N_{i}^{M}(c):=\sum_{j=i}^{M} c_{i, j} \quad \text { and } \quad \mathcal{N}_{p}^{M}:=\sum_{i=1}^{p} N_{i}^{M} .
$$

For $t, \tau>0$ we have, after some algebraic manipulations,

$$
\begin{aligned}
& \mathcal{N}_{p}^{M}(c(t+\tau))-\mathcal{N}_{p}^{M}(c(t))= \\
& =\left(c_{1,1}(t+\tau)-c_{1,1}(t)\right)+\sum_{i=2}^{p} \sum_{j=i}^{M}\left(c_{i, j}(t+\tau)-c_{i, j}(t)\right) \\
& =-\int_{t}^{t+\tau} \ell_{1}^{2} c_{1,1}^{2}-\int_{t}^{t+\tau} \ell_{1} c_{1,1}\left(\sum_{i=2}^{\infty} \sum_{j=i}^{\infty} \ell_{i} c_{i, j}+\sum_{i=2}^{p} \ell_{i} c_{i, M}\right)+\int_{t}^{t+\tau} Q^{p, M}(c)
\end{aligned}
$$


where

$$
\begin{aligned}
Q^{p, M}(c)= & \sum_{i=3}^{p} \sum_{j=2 i-2}^{M} \sum_{m=\left\lfloor\frac{j+1}{2}\right\rfloor}^{j-i+1} \ell_{i-1}^{2} c_{i-1, m} c_{i-1, j-m} \\
& +\sum_{i=3}^{p} \sum_{j=i+2}^{M} \sum_{m=i}^{j-2} \sum_{k=2}^{(i-1) \wedge(j-m)} \ell_{i} \ell_{k} c_{i, m} c_{k, j-m} \\
& -\sum_{i=2}^{p} \sum_{j=i}^{M} \sum_{m=i}^{\infty} \ell_{i}^{2} c_{i, j} c_{i, m} \\
& -\sum_{i=2}^{p} \sum_{j=i}^{M} \ell_{i}^{2} c_{i, j}^{2} \\
& -\sum_{i=2}^{p} \sum_{j=i}^{M} \sum_{\substack{k=2 \\
k \neq i}}^{\infty} \sum_{m=k}^{\infty} \ell_{i} \ell_{k} c_{i, j} c_{k, m} .
\end{aligned}
$$

We need to write $Q^{p, M}(c)$ in a way that its sign can be clearly determined. Since our goal is to let $M \rightarrow \infty$ we will always consider $M$ as so large as required for the algebraic manipulations we will perform to make sense. Some of these manipulations will be quite close to those presented in the proof of Proposition 3, and so we will now just outline the main steps.

Starting with (43), and repeating the steps that produced (23) to (18) we now obtain

$$
\begin{aligned}
& \sum_{i=3}^{p} \sum_{j=2 i-2}^{M} \sum_{m=\left\lfloor\frac{j+1}{2}\right\rfloor}^{j-i+1} \ell_{i-1}^{2} c_{i-1, m} c_{i-1, j-m}= \\
& \quad=\sum_{i=2}^{p-1} \sum_{j=\left\lfloor\frac{M+1}{2}\right\rfloor}^{M-i} \sum_{m=i}^{M-j} \ell_{i}^{2} c_{i, j} c_{i, m}+\sum_{i=2}^{p-1} \sum_{j=i}^{\left\lfloor\frac{M-1}{2}\right\rfloor} \sum_{m=j}^{\left\lfloor\frac{M-1}{2}\right\rfloor} \ell_{i}^{2} c_{i, j} c_{i, m}
\end{aligned}
$$

For the expression (44) we repeat the procedure that led from (19) to (25) and have now

$$
\begin{aligned}
& \sum_{i=3}^{p} \sum_{j=i+2}^{M} \sum_{m=i}^{j-2} \sum_{k=2}^{(i-1) \wedge(j-m)} \ell_{i} \ell_{k} c_{i, m} c_{k, j-m}= \\
& =\sum_{i=2}^{p-1} \sum_{j=i}^{p-1} \sum_{k=j+1}^{p} \sum_{m=k}^{M-j} \ell_{i} \ell_{k} c_{i, j} c_{k, m}+\sum_{i=3}^{p} \sum_{m=i}^{M-i} \sum_{j=i}^{M-m} \sum_{k=2}^{i-1} \ell_{i} \ell_{k} c_{i, j} c_{k, m}
\end{aligned}
$$

Changing the order of summation $\sum_{j} \sum_{k}$ to $\sum_{k} \sum_{j}$ in the first multiple sum of (49) and, in the second multiple sum, first transforming it from the form $\sum_{i} \sum_{m} \sum_{j} \sum_{k}$ to $\sum_{k} \sum_{i} \sum_{m} \sum_{j}$ and then changing notation $(k, m, i, j) \mapsto$ 
$(i, j, k, m)$, we can sum the resulting terms to write (49) in the simpler form

$$
\sum_{i=2}^{p-1} \sum_{k=i+1}^{p} \sum_{j=i}^{M-k} \sum_{m=k}^{M-j} \ell_{i} \ell_{k} c_{i, j} c_{k, m}
$$

The terms (45) and (46) can be easily transformed:

$$
\begin{aligned}
& -\sum_{i=2}^{p} \sum_{j=i}^{M} \sum_{m=i}^{\infty} \ell_{i}^{2} c_{i, j} c_{i, m}-\sum_{i=2}^{p} \sum_{j=i}^{M} \ell_{i}^{2} c_{i, j}^{2}= \\
& =-\sum_{i=2}^{p} \sum_{j=i}^{M} \sum_{m=i}^{M} \ell_{i}^{2} c_{i, j} c_{i, m}-\sum_{i=2}^{p} \sum_{j=i}^{M} \sum_{m=M+1}^{\infty} \ell_{i}^{2} c_{i, j} c_{i, m}-\sum_{i=2}^{p} \sum_{j=i}^{M} \ell_{i}^{2} c_{i, j}^{2} \\
& =-2 \sum_{i=2}^{p} \sum_{j=i}^{M} \sum_{m=j}^{M} \ell_{i}^{2} c_{i, j} c_{i, m}-\sum_{i=2}^{p} \sum_{j=i}^{M} \sum_{m=M+1}^{\infty} \ell_{i}^{2} c_{i, j} c_{i, m}
\end{aligned}
$$

where in the last equality we made use of the symmetry of the summand in the $(j, m)$-square $[i, M]^{2}$. The first multiple sum of (51) can be transformed into

$$
\begin{aligned}
& -2 \sum_{i=2}^{p} \sum_{j=i}^{M} \sum_{m=j}^{M} \ell_{i}^{2} c_{i, j} c_{i, m}= \\
& =-\sum_{i=2}^{p-1} \sum_{j=i}^{M} \sum_{m=j}^{M} \ell_{i}^{2} c_{i, j} c_{i, m}-\sum_{i=2}^{p} \sum_{j=i}^{M} \sum_{m=j}^{M} \ell_{i}^{2} c_{i, j} c_{i, m}-\sum_{j=p}^{M} \sum_{m=j}^{M} \ell_{p}^{2} c_{p, j} c_{p, m}
\end{aligned}
$$

Adding the first term in the right hand side of (52) with the second multiple sum of (48) gives

$$
-\sum_{i=2}^{p-1} \sum_{m=\left\lfloor\frac{M+1}{2}\right\rfloor}^{M} \sum_{j=i}^{m} \ell_{i}^{2} c_{i, j} c_{i, m}
$$

Finally, for (47), we can write

$$
\begin{aligned}
& -\sum_{i=2}^{p} \sum_{j=i}^{M} \sum_{\substack{k=2 \\
k \neq i}}^{\infty} \sum_{m=k}^{\infty} \ell_{i} \ell_{k} c_{i, j} c_{k, m}= \\
& =-\sum_{i=2}^{p} \sum_{j=i}^{M} \sum_{k=i+1}^{\infty} \sum_{m=k}^{\infty} \ell_{i} \ell_{k} c_{i, j} c_{k, m}-\sum_{i=3}^{p} \sum_{j=i}^{M} \sum_{k=2}^{i-1} \sum_{m=k}^{\infty} \ell_{i} \ell_{k} c_{i, j} c_{k, m} \\
& =-\sum_{i=2}^{p} \sum_{j=i}^{M} \sum_{k=i+1}^{\infty} \sum_{m=k}^{\infty} \ell_{i} \ell_{k} c_{i, j} c_{k, m}-\sum_{i=2}^{\infty} \sum_{j=i}^{\infty} \sum_{k=i+1}^{p} \sum_{m=k}^{M} \ell_{i} \ell_{k} c_{i, j} c_{k, m}
\end{aligned}
$$

where the last equality was obtained by some obvious changes in the order of summation until the second multiple sum becomes written in the order $\sum_{k} \sum_{m} \sum_{i} \sum_{j}$ followed by an appropriate renaming of the variables. 
Putting together (48), (50), (51), (52), and (54) we can write $Q^{p, M}(c)$ in the following form

$$
\begin{aligned}
Q^{p, M}(c)= & -\sum_{i=2}^{p} \sum_{j=i}^{M} \sum_{m=j}^{M} \ell_{i}^{2} c_{i, j} c_{i, m}-\sum_{j=p}^{M} \sum_{m=j}^{M} \ell_{p}^{2} c_{p, j} c_{p, m} \\
& -\sum_{i=2}^{p} \sum_{j=i}^{M} \sum_{k=i+1}^{\infty} \sum_{m=k}^{\infty} \ell_{i} \ell_{k} c_{i, j} c_{k, m} \\
& -\sum_{i=2}^{p-1}\left(\sum_{m=\left\lfloor\frac{M+1}{2}\right\rfloor}^{M} \sum_{j=i}^{m}-\sum_{j=\left\lfloor\frac{M+1}{2}\right\rfloor}^{M-i} \sum_{m=i}^{M-j}\right) \ell_{i}^{2} c_{i, j} c_{i, m} \\
& -\sum_{i=2}^{p-1} \sum_{k=i+1}^{p}\left(\sum_{j=i}^{\infty} \sum_{m=k}^{M}-\sum_{j=i}^{M-k} \sum_{m=k}^{M-j}\right) \ell_{i} \ell_{k} c_{i, j} c_{k, m}
\end{aligned}
$$

We now need to establish the signs of (55) and (56). In the case of (55) first change the notation $(j, m) \mapsto(m, j)$ in the second double sum and then observe that

$$
\begin{gathered}
\left\{(j, m):\left\lfloor\frac{M+1}{2}\right\rfloor \leq m \leq M-i, i \leq j \leq M-m\right\} \subset \\
\subset \quad\left\{(j, m):\left\lfloor\frac{M+1}{2}\right\rfloor \leq m \leq M, i \leq j \leq m\right\} .
\end{gathered}
$$

Since the summand in (55) is nonnegative we conclude that the full term (55) has a negative contribution to $Q^{p, M}(c)$. For (56) just observe that

$$
\begin{gathered}
\{(j, m): i \leq j \leq M-k, k \leq m \leq M-j\} \subset \\
\subset \quad\{(j, m): i \leq j<+\infty, k \leq m \leq M\},
\end{gathered}
$$

and so, again, the contribution to $Q^{p, M}(c)$ is negative. This allow us to conclude that, for all $p, M$, and nonnegative $c$, we have $Q^{p, M}(c) \leq 0$. Hence, by $(42), \mathcal{N}_{p}^{M}$ is nonincreasing along solutions. As a consequence, $\mathcal{N}_{p}=\lim _{M \rightarrow \infty} \mathcal{N}_{p}^{M}$ is also nonincreasing along solutions. Since both $\mathcal{N}_{p}^{M}(c(t))$ and $\mathcal{N}_{p}(c(t))$ are bounded below by zero we conclude that they converge to some nonnegative real as $t \rightarrow \infty$. From this it follows that $N_{p}(c(t))=\mathcal{N}_{p}(c(t))-\mathcal{N}_{p-1}(c(t))$ also converge to some nonnegative constant $\gamma_{p}$ as $t \rightarrow \infty$. To determine the value of $\gamma_{p}$ we need to have an equation for the time evolution of $N_{p}(c(t))$. This will be deduced from the equation (42). Write $N_{p}$ as

$$
N_{p}=\lim _{M \rightarrow \infty} N_{p}^{M}=\lim _{M \rightarrow \infty}\left(\mathcal{N}_{p}^{M}-\mathcal{N}_{p-1}^{M}\right) .
$$


We can use (42) to compute

$$
\begin{aligned}
& N_{p}^{M}(c(t+\tau))-N_{p}^{M}(c(t))= \\
& =\left(\mathcal{N}_{p}^{M}(c(t+\tau))-\mathcal{N}_{p-1}^{M}(c(t+\tau))\right)+\left(\mathcal{N}_{p}^{M}(c(t))-\mathcal{N}_{p-1}^{M}(c(t))\right) \\
& =\left(\mathcal{N}_{p}^{M}(c(t+\tau))-\mathcal{N}_{p}^{M}(c(t))\right)+\left(\mathcal{N}_{p-1}^{M}(c(t))-\mathcal{N}_{p-1}^{M}(c(t))\right) \\
& =-\int_{t}^{t+\tau} \ell_{1} \ell_{p} c_{1,1} c_{p, M}+\int_{t}^{t+\tau}\left(Q^{p, M}(c)-Q^{p-1, M}(c)\right) \\
& =-\int_{t}^{t+\tau}\left(2 \sum_{j=p}^{M} \sum_{m=j}^{M} \ell_{p}^{2} c_{p, j} c_{p, m}+\sum_{j=p}^{M} \sum_{k=p+1}^{\infty} \sum_{m=k}^{\infty} \ell_{p} \ell_{k} c_{p, j} c_{k, m}-\right. \\
& \left.-\sum_{j=p-1}^{M} \sum_{m=j}^{M} \ell_{p-1}^{2} c_{p-1, j} c_{p-1, m}\right)- \\
& -\int_{t}^{t+\tau}\left[\ell_{1} \ell_{p} c_{1,1} c_{p, M}+\right. \\
& +\left(\sum_{j=\left\lfloor\frac{M+1}{2}\right\rfloor}^{M} \sum_{m=\left\lfloor\frac{M}{2}\right\rfloor+1}^{j}+\sum_{m=p-1}^{\left\lfloor\frac{M}{2}\right\rfloor} \sum_{j=M-m+1}^{M}\right) \ell_{p-1}^{2} c_{p-1, j} c_{p-1, m}+ \\
& \left.+\sum_{i=2}^{p-1}\left(\sum_{j=i}^{\infty} \sum_{m=M-i+1}^{M}+\sum_{m=p}^{M-i} \sum_{j=M-m}^{\infty}\right) \ell_{i} \ell_{p} c_{i, j} c_{p, m}\right]
\end{aligned}
$$

When $M \rightarrow \infty$ the second integral converges to zero and we obtain the evolution equation

$$
\begin{aligned}
N_{p}(c(t+\tau))-N_{p}(c(t))= & \\
=-\int_{t}^{t+\tau}\left(2 \sum_{j=p}^{\infty} \sum_{m=j}^{\infty} \ell_{p}^{2} c_{p, j} c_{p, m}\right. & +\sum_{j=p}^{\infty} \sum_{k=p+1}^{\infty} \sum_{m=k}^{\infty} \ell_{p} \ell_{k} c_{p, j} c_{k, m}- \\
& \left.-\sum_{j=p-1}^{\infty} \sum_{m=p-1}^{j} \ell_{p-1}^{2} c_{p-1, j} c_{p-1, m}\right)
\end{aligned}
$$

Observing that

$$
\begin{aligned}
2 \sum_{j=p}^{\infty} \sum_{m=j}^{\infty} \ell_{p}^{2} c_{p, j} c_{p, m} & =\sum_{j=p}^{\infty} \sum_{m=p}^{\infty} \ell_{p}^{2} c_{p, j} c_{p, m}+\sum_{j=p}^{\infty} \ell_{p}^{2} c_{p, j}^{2} \\
& =\ell_{p}^{2} N_{p}^{2}+\sum_{j=p}^{\infty} \ell_{p}^{2} c_{p, j}^{2} \\
& \geq \ell_{p}^{2} N_{p}^{2}
\end{aligned}
$$


and

$$
\begin{aligned}
\sum_{j=p-1}^{\infty} \sum_{m=p-1}^{j} \ell_{p-1}^{2} c_{p-1, j} c_{p-1, m} & =\ell_{p-1}^{2} \sum_{j=p-1}^{\infty} c_{p-1, j} \sum_{m=p-1}^{j} c_{p-1, m} \\
& \leq \ell_{p-1}^{2} \sum_{j=p-1}^{\infty} c_{p-1, j} \sum_{m=p-1}^{\infty} c_{p-1, m} \\
& =\ell_{p-1}^{2} N_{p-1}^{2}
\end{aligned}
$$

we can estimate the right hand side of (58) to obtain

$$
N_{p}(c(t+\tau))-N_{p}(c(t)) \leq \int_{t}^{t+\tau}\left(\ell_{p-1}^{2} N_{p-1}^{2}-\ell_{p}^{2} N_{p}^{2}\right)
$$

We can now apply the induction method: suppose $N_{p-1}(c(t)) \rightarrow 0$ as $t \rightarrow \infty$. We have already proved that $N_{p}(c(t)) \rightarrow \gamma_{p} \geq 0$ as $t \rightarrow \infty$. Suppose $\gamma_{p}>0$. As in the proof of Proposition 7, we now take limits as $t \rightarrow \infty$ in (61) and obtain $0 \leq-\frac{1}{2} \ell_{p}^{2} \gamma_{p}^{2} \tau$, which contradicts $\tau>0$. This concludes the proof.

\section{The Simplified Order Equations}

\subsection{The Local Interaction System}

In the previous section we proved that the total number of clusters of a given fixed order $p$, denoted by $N_{p}$, tend to zero as $t \rightarrow \infty$. As pointed out in the Introduction, these quantities are particularly interesting in certain applications and can be viewed as a kind of mesoscopic scale description of the system, somewhere between the microscopic description provided by the individual cluster concentrations $c_{i, j}$ and the macroscopic quantities describing the total mass or the total number of clusters at a given time. Unfortunately, the evolution equation for $N_{p}$ given in (58) is not closed, and so the full description of the behaviour of $N_{p}$ has the same order of difficulty as that of the original microscopic system. However, an approximate description is possible by using the estimates (59) and (60). The resulting differential inequality (61) seems a lot simpler that (58) and, although it does not give us precise information about the behaviour of the quantities $N_{p}$, at least it is a lot more amenable to analysis.

We go a step further in the simplification procedure and consider the equation obtained by considering the equality sign in (61). From now on we shall also put $\ell_{i} \equiv 1$. With these assumptions we can derive a closed systems for the quantities $N_{p}$. For $p \geq 2$ we will have the equations $\dot{N}_{p}=N_{p-1}^{2}-N_{p}^{2}$. For $p=1$, by (2), $\dot{N}_{1}=\dot{c}_{1,1}=-2 N_{1}^{2}-N_{1} \sum_{i=2}^{\infty} N_{i}=-N_{1}^{2}-N_{1} N_{0}$, where $N_{0}:=\sum_{i=1}^{\infty} N_{i}$. We can derive an equation for $N_{0}$ using the previous two equations: $\dot{N}_{0}=\sum_{i=1}^{\infty} \dot{N}_{i}=$ $\dot{N}_{1}+\sum_{i=2}^{\infty} \dot{N}_{i}=-N_{1}^{2}-N_{1} N_{0}+\sum_{i=2}^{\infty}\left(N_{i-1}^{2}-N_{i}^{2}\right)=-N_{1} N_{0}$. Hence, as a first approximation to the study of the quantities $N_{p}$ we shall consider the closed 
system

$$
\left\{\begin{array}{l}
\dot{N}_{0}=-N_{1} N_{0} \\
\dot{N}_{1}=-N_{1} N_{0}-N_{1}^{2} \\
\dot{N}_{p}=N_{p-1}^{2}-N_{p}^{2}, \quad p \geq 2 .
\end{array}\right.
$$

It is interesting to observe that a system like the one for $N_{p}, p \geq 2$, also arises as a crude model for fracture dynamics [22]. Also worth mentioning is the fact that system (62), when interpreted in the context of river network studies, is a model of a "structurally Hortonian" network, ie, one in which any $p$ order cluster increases only through the contribution of $p-1$ order clusters $[8, \mathrm{pp}$ 250].

Besides the fact of being closed, this system has the additional advantage that the coupling between the variables $N_{p}$ is local. Both these facts will greatly simplify our study. Clearly, the method used in the last section to get convergence to zero can again be applied without modification to system (62) in order to conclude the following

Proposition 9 Let $N^{\text {in }}=\left(N_{p}^{\text {in }}\right)$ be an arbitrary nonnegative initial data, and let $N=\left(N_{p}\right)$ be the solution of (62) with initial data $N^{\text {in }}$. Then, as $t \rightarrow+\infty$, we have

$$
N_{p}(t) \longrightarrow N_{p}^{\infty}:=N_{0}^{\text {in }} e^{-N_{1}^{\text {in }} / N_{0}^{\text {in }}} \delta_{p=0} .
$$

Proof: We start by noticing that in (62) the subsystem for the variables $N_{0}$ and $N_{1}$ can be studied separately from the rest. So, let us consider the subsystem

$$
\left\{\begin{array}{l}
\dot{N}_{0}=-N_{1} N_{0} \\
\dot{N}_{1}=-N_{1} N_{0}-N_{1}^{2} .
\end{array}\right.
$$

Observe that solutions with nonnegative initial data are globally defined in $\mathbb{R}_{+}$ since the boundary of $\mathbb{R}_{+}^{2}$ is invariant for (64) and solutions of system (64) have their components decreasing. Phase plane analysis imediately imply that $N_{1}(t) \rightarrow 0$ as $t \rightarrow+\infty$, and also that $N_{0}(t)$ converges to some nonnegative constant.

From this result for the behaviour of $N_{1}(t)$, it easily follows that $N_{p}(t) \rightarrow 0$ as $t \rightarrow+\infty$, for all $p \geq 1$, by an application of the arguments already used in the previous Section.

We are only left to identify the nonnegative value of the limite of $N_{0}(t)$ when $t \rightarrow+\infty$. Using the strict monotonicity of $N_{0}(t)$ we can consider $N_{1}$ as a function of $N_{0}$ and obtain

$$
\frac{d N_{1}}{d N_{0}}=1+\frac{N_{1}}{N_{0}}
$$

This homogeneous differential equation can be solved by a standard change of variables and we get

$$
N_{1}=\left(C^{\text {in }}+\log N_{0}\right) N_{0}
$$

where $C^{\text {in }}:=\frac{N_{1}^{\text {in }}}{N_{0}^{\text {in }}}-\log N_{0}^{\text {in }}$. After substitution, the equation for $N_{0}$ becomes

$$
\dot{N}_{0}=-\left(C^{\text {in }}+\log N_{0}\right) N_{0}^{2}
$$


and elementary qualitative analysis allow us to conclude that

$$
N_{0}(t) \longrightarrow e^{-C^{\text {in }}}=N_{0}^{\text {in }} e^{-N_{1}^{\text {in }} / N_{0}^{\text {in }}}, \text { as } t \rightarrow+\infty,
$$

as we wanted to prove.

In addition, for this system, we can probe further into the details of the asymptotic behaviour, as we shall present in the remaining of this section.

\subsubsection{Unimodality of Solutions}

In applications (in the case of the morphology of river networks, but also in others instances) it is particularly important to consider initial data consisting only of monomers.

A numerical solution of a truncation of (62) to fifty equations, and with these type of initial conditions, is shown in Figure 5. The plot suggests a number of features of the behaviour of the solutions that deserve attention. One is the unimodality of solutions, which we prove next. Another is the details of the large-time asymptotics, which will be treated later.

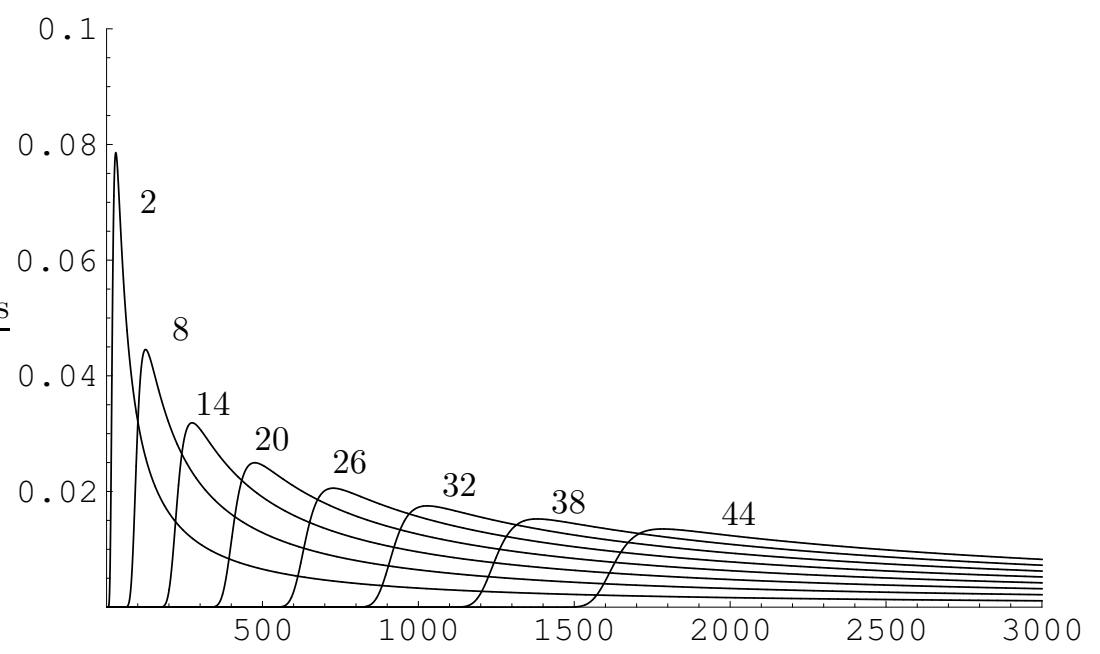

Figure 5: Numerical solution of a truncation of (62), with monomeric initial condition. The plots of $N_{p}$ for $p=2,8,14,20,26,32,38,44$ are shown.

We now prove the following result

Proposition 10 Let $N=\left(N_{p}\right)$ be the solution of (62) with monomer initial data $N_{p}(0)=A \delta_{p \in\{0,1\}}$, for some $A>0$. Then, all components of the solution vector $N$ are unimodal.

We will actually prove a bit more than stated above, by establishing that the time $\tau_{p}$ at which $N_{p}$ attains its (unique) maximum satisfies $0 \leq \tau_{p}<$ $\tau_{p+1}, \forall p \geq 1$. 
In order to prove Proposition 10 we need the following Lemma that allow us to control the behaviour of $N_{p}(t)$ for $t$ sufficiently close to 0 .

Lemma 1 With the conditions of Proposition 10, the first non-zero derivative of $N_{p}$ at $t=0$ is of order $2^{p-1}-1$, and is positive, for all $p \geq 1$.

Proof: From $\dot{N}_{p}=N_{p-1}^{2}-N_{p}^{2}$ we obtain, by the Leibnitz rule

$$
N_{p}^{(j)}=\sum_{r=0}^{j-1}\left(\begin{array}{c}
j-1 \\
r
\end{array}\right) N_{p-1}^{(j-1-r)} N_{p-1}^{(r)}-\sum_{r=0}^{j-1}\left(\begin{array}{c}
j-1 \\
r
\end{array}\right) N_{p}^{(j-1-r)} N_{p}^{(r)} .
$$

Let us proceed by induction. For $p=1$ we have $2^{p-1}-1=0$ and the result is true since $N_{1}^{(0)}(0)=N_{1}(0)=A>0$. Assume the result holds for $N_{p-1}$. Then, by (66), if the order of the first nonzero derivative of $N_{p-1}$ at $t=0$ is $(p-1)^{*}:=2^{(p-1)-1}-1$, then the first nonzero contribution to (66) must be due to the term

$$
N_{p-1}^{\left((p-1)^{*}\right)}(0) N_{p-1}^{\left((p-1)^{*}\right)}(0)
$$

and so we must have $j-1-(p-1)^{*}=(p-1)^{*}$ which implies that $j=$ $2(p-1)^{*}+1=2\left(2^{p-2}-1\right)+1=2^{p-1}-1$. Furthermore, the second sum in (66) is always zero at $t=0$ for $j \leq 2^{p-1}-1$, since if it were nonzero for some $j^{*} \leq 2^{p-1}-1$, then the product

$$
N_{p}^{\left(j^{*}-1-r\right)}(0) N_{p}^{(r)}(0)
$$

would have to be nonzero for some $r<j^{*}$. Proceeding down from here we would obtain $N_{p}^{(0)}(0)=N_{p}(0)>0$, which contradicts the assumption on the initial data. This proves the Lemma.

Proof of Proposition 10: We start by the function $N_{0}$. Since $\dot{N}_{0}=-N_{1} N_{0}<0$ we conclude that $N_{0}$ is strictly decreasing and hence unimodal with maximum at $t=\tau_{0}=0$.

For the functions $N_{p}$ we shall proceed by induction. To prove the assertion for $N_{p}$ we shall need to use the behaviour of $N_{p-1}$ and $N_{p-2}$, and so we need to start by looking at the behaviour of $N_{1}$ and $N_{2}$. For $N_{1}$ the situation is the same as with $N_{0}: \dot{N}_{1}=-N_{1}^{2}-N_{1} N_{0}<0 \Rightarrow N_{1}$ is strictly decreasing, and so $N_{1}$ is unimodal with maximum at $t=\tau_{1}=0$. Consider now the case of $N_{2}$ : all stationary points of $N_{2}$, and hence all maxima, occur when $0=\dot{N}_{2}=$ $N_{1}^{2}-N_{2}^{2} \Leftrightarrow N_{2}=N_{1}$ and at those points it holds

$$
\begin{aligned}
\ddot{N}_{2} & =2 N_{1} \dot{N}_{1}-2 N_{2} \dot{N}_{2} \\
& =-2 N_{1}^{2}\left(N_{1}+N_{0}\right) \\
& <0,
\end{aligned}
$$

from which we conclude that all stationary points are maxima, and so it can exist only one, and since $N_{2}(0)=0=\lim _{t \rightarrow+\infty} N_{2}(t)$ and $N_{2}(t)>0$ for $t>0$, we conclude that there exists exactly one maximum and hence $N_{2}$ is unimodal. 
We now proceed by induction: assume $N_{p-2}$ and $N_{p-1}$ are both unimodal with maxima at $t=\tau_{p-2}$ and $t=\tau_{p-1}$ respectively, and $\tau_{p-2}<\tau_{p-1}$. We shall prove that $N_{p}$ is also unimodal with $\tau_{p}>\tau_{p-1}$, where $\tau_{p}$ is the time at which $N_{p}$ attains its maximum.

Let $t=\tau$ be the smallest positive time for which $\dot{N}_{p}=0$. Thus, at $\tau$ we have $N_{p-1}(\tau)=N_{p}(\tau)$. We first prove that $\tau>\tau_{p-1}$.

Assume $\tau<\tau_{p-1}$. From the definition of $\tau$ and the unimodality of $N_{p-1}$ it follows that $N_{p-1}(t)<N_{p}(t)$ for all $t<\tau$ sufficiently close to $\tau$. But, by Lemma 66 , for all $t>0$ sufficiently small, $N_{p-1}(t)>N_{p}(t)$, and so, by continuity, there exists $\tilde{\tau} \in(0, \tau)$ such that $N_{p-1}(\tilde{\tau})=N_{p}(\tilde{\tau})$ which implies $\dot{N}_{p}(\tilde{\tau})=0$, contradictiong the assumption that $\tau$ was the smallest such (positive) time.

Assume now that $\tau=\tau_{p-1}$. Thus $N_{p-2}(\tau)=N_{p-1}(\tau)=N_{p}(\tau)$ and so, at $t=\tau$,

$$
\begin{aligned}
& \left(N_{p-1}-N_{p}\right)^{\cdot}=\dot{N}_{p-1}-\dot{N}_{p}=N_{p-2}^{2}-2 N_{p-1}^{2}+N_{p}^{2}=0 \\
& \left(N_{p-1}-N_{p}\right)^{\cdot}=2 N_{p-2} \dot{N}_{p-2}-4 N_{p-1} \dot{N}_{p-1}+2 N_{p} \stackrel{N}{p}_{p}=2 N_{p-2} \dot{N}_{p-2}
\end{aligned}
$$

since at $t=\tau=\tau_{p-1}$ both $\dot{N}_{p}$ and $\dot{N}_{p-1}$ are zero. From the induction hypothesis, the inequality $\tau_{p-2}<\tau$, and the unimodality of $N_{p-2}$, we conclude that $\dot{N}_{p-2}(\tau)<0$ and so $\left(N_{p-1}-N_{p}\right)^{*}(\tau)<0$. In the other hand, the initial condition and Lema 66 imply $\left(N_{p-1}-N_{p}\right)(0)=0$ and $\left(N_{p-1}-N_{p}\right)(t)>0$ for sufficiently small $t>0$, and, by continuity and the definition of $\tau$, the inequality holds for all $t \in(0, \tau)$. But then $\left.\left(N_{p-1}-N_{p}\right)\right|_{(0, \tau]}(t)$ has a minimum at $t=\tau$, contradicting the inequality for the second derivative obtained above.

This allow us to conclude that $\tau>\tau_{p-1}$. But then, at $t=\tau$, we have

$$
\begin{aligned}
\ddot{N}_{p} & =2 N_{p-1} \dot{N}_{p-1}-2 N_{p} \dot{N}_{p} \\
& =2 N_{p-1}\left(N_{p-1}^{2}-N_{p}^{2}\right) \\
& <0
\end{aligned}
$$

which implies that the stationary point must be a maximum of $N_{p}$. By definition of $\tau$, all other stationary points of $N_{p}$ must occur at times $t=\tilde{\tau}>\tau>\tau_{p-1}$ and the same conclusion holds: they must be maxima. This implies there is only one stationary point and so $N_{p}$ is unimodal with its maximum at $\tau_{p}:=\tau>\tau_{p-1}$, as we wanted to prove.

Without much extra effort we can prove the sequence $\left(\tau_{p}\right)$ converge to $+\infty$.

Proposition 11 With the conditions of Proposition 10, let $\tau_{p}$ be the value of $t$ for which $N_{p}(t)$ attains its maximum. The sequence $\left(\tau_{p}\right)$ is strictly increasing and convergent to $+\infty$.

Proof: The monotonicity was already established in the proof of Proposition 10. Also in that proof, we concluded that, for $p \geq 2$, the maximum of $N_{p}$ occurs at the intersection of $N_{p}$ and $N_{p-1}$ and, furthermore, the intersection is transversal and $N_{p}>N_{p-1}$ after the intersection point. This shall now be crucial. 
Suppose $\tau_{p} \not \rightarrow+\infty$. Being monotonic, the sequence $\left(\tau_{p}\right)$ must converge to some $\tau \in \mathbb{R}^{+}$. Let $\varepsilon>0$ be arbitrary. Let $p=p(\varepsilon)$ be the smallest integer such that $\tau_{p}>\tau-\varepsilon$. Then, since $\tau_{j} \uparrow \tau$, it follows that, for all $t \geq \tau$ and $j>p$, we have $N_{j}(t)>N_{p}(t)$ and so the function

$$
N_{0}(t)=\sum_{j=1}^{\infty} N_{j}(t)
$$

blows up to $+\infty$ as $t \uparrow \tau$, which contradicts the fact that $N_{0}$ is strictly decreasing in $(0, \tau)$ since for this region of times $N_{0}$ must satisfy the first equation in system (62). This concludes the proof.

Another easy consequence of the above results is the behaviour of the maximum of $N_{p}(t)$ as a function of $p$, which is presented next

Proposition 12 With the conditions of Proposition 11, the sequence $\left(N_{p}^{\max }\right)$, where $N_{p}^{\max }:=N_{p}\left(\tau_{p}\right)$, is strictly decreasing and convergent to zero.

Proof: Since all $N_{p}$ are unimodal and, for a given $p \geq 2$, the function $N_{p}(t)$ attains its maximum at the value of $t=\tau_{p}$ where $N_{p}$ and $N_{p-1}$ intersect, we conclude that

$$
N_{p}^{\max }=N_{p}\left(\tau_{p}\right)=N_{p-1}\left(\tau_{p}\right)<N_{p-1}\left(\tau_{p-1}\right)=N_{p-1}^{\max } .
$$

This, together with the asymptotic behaviours $\tau_{p} \rightarrow+\infty$, and $N_{p}(t) \rightarrow 0$ as $t \rightarrow \infty$ allow us to obtain the result.

\subsubsection{Large-time asymptotics}

We now give the large-time asymptotic decay of the quantities $N_{p}(t)$, starting with the closed two-dimensional subsystem for $\left(N_{0}, N_{1}\right)(64)$. The leading order form of convergence is given by (63): since $N_{1} \rightarrow 0$ and $N_{0} \rightarrow N_{0}^{\infty} \neq 0$, in the large time limit, the second of (64) can be approximated by $\dot{N}_{1}=-N_{1} N_{0}^{\infty}$. On solving this, the solution can be substituted into (65) to find the corresponding perturbation to $N_{0}$. We find that

$$
N_{1} \sim n_{1} e^{-N_{0}^{\infty} t}, \quad N_{0} \sim N_{0}^{\infty}+n_{1} e^{-N_{0}^{\infty} t} \quad \text { as } t \rightarrow \infty .
$$

Now that the large-time asymptotics of $N_{1}$ are known, corresponding results for $N_{2}, N_{3}, \ldots$ can be found sequentially. The equation for $N_{2}$ is then

$$
\dot{N}_{2}=-N_{2}^{2}+n_{1}^{2} e^{-2 N_{0}^{\infty} t}
$$

which is solved by

$$
N_{2}=n_{1} e^{-N_{0}^{\infty} t} \frac{\left.K_{1}\left(n_{1} e^{-N_{0}^{\infty} t} / N_{0}^{\infty}\right)-C I_{1}\left(n_{1} e^{-N_{0}^{\infty} t} / N_{0}^{\infty}\right)\right)}{C I_{0}\left(n_{1} e^{-N_{0}^{\infty} t} / N_{0}^{\infty}\right)+K_{0}\left(n_{1} e^{-N_{0}^{\infty} t} / N_{0}^{\infty}\right)} .
$$

At large times, this asymptotes to $N_{2} \sim 1 / t$. Thus $\left\{N_{p}\right\}_{p=2}^{\infty}$ all follow the expected $1 / t$ large time behaviour (though note that $N_{1}$ does not). The form 
of the factor can also be found, by assuming $N_{p}(t) \sim n_{p} / t$ as $t \rightarrow \infty$, we find that the coefficient $n_{p}$ satisfies $-n_{p}=n_{p-1}^{2}-n_{p}^{2}$, and thus

$$
n_{p}=\frac{1}{2}\left(1+\sqrt{1+4 n_{p-1}^{2}}\right) .
$$

Analysing this first order map, we find $n_{p}>n_{p-1}$ for all $p$ and, as $p \rightarrow \infty$, $n_{p} \sim n_{p-1}+\frac{1}{2}$. Thus in the large $p$ limit we have $n_{p} \sim \frac{1}{2} p+n_{0}$ for some constant $n_{0}$. To summarise as $t \rightarrow \infty$, we have $N_{p}(t) \sim n_{p} / t$ as $t \rightarrow \infty$ for $p>1$; and for large $p, N_{p}(t) \sim p / 2 t$.

\subsection{The Non-Local Interaction System}

We now move from the locally interacting system (62) to the more accurate approximation

$$
\left\{\begin{array}{l}
\dot{N}_{1}=-N_{1}^{2}-N_{1} N_{0} \\
\dot{N}_{p}=N_{p-1}^{2}-N_{p}\left(N_{p}+N_{p+1}+N_{p+2}+\ldots\right), \quad p \geq 2 .
\end{array}\right.
$$

where $N_{0}$ is defined by $N_{0}:=\sum_{i=1}^{\infty} N_{i}$ as earlier.

System (67) is obtained from (58) by keeping the triple sum in its right hand side; for $\ell_{j} \equiv 1$ this sum is exactly equal to $N_{p}\left(N_{p}+N_{p+1}+N_{p+2}+\ldots\right)$. Solutions to $(67)$ are thus expected to be a better approximation to the true time evolution of $N_{p}(t)$. The coarse asymptotic behaviour of solutions as $t \rightarrow \infty$ is easily concluded:

Proposition 13 All nonnegative solutions to (67) converge to zero as $t \rightarrow+\infty$.

Proof: The proof is elementary: for $N_{1}$ we need only to observe that nonnegative solutions satisfy to $\dot{N}_{1} \leq-2 N_{1}^{2}$ in order to conclude the result. For $N_{p}$ with $p \geq 2$ note that

$$
\dot{N}_{1}=N_{p-1}^{2}-N_{p}\left(N_{p}+N_{p+1}+N_{p+2}+\ldots\right) \leq N_{p-1}^{2}-N_{p}^{2},
$$

and proceed by induction.

The inequality in the proof just concluded imply that solutions to this system will converge faster to zero than the corresponding ones of system (62). Evidence of this is clear in the plots of numerical solutions such as the one presented in Figure 6 .

We note that solutions are also unimodal for monomeric initial data. This result, that was proved in Proposition 10 for the local interacting system (62), seems to be a lot harder to prove now, due, mainly, to the dificulty of controling all the remaining components of the solution vector when studing a given fixed component.

Somewhat surprisingly, however, a rather detailed, albeit formal, study of the details of the long time behaviour is possible in the present case, shedding some light into the possible occurence of similarity behaviour in solutions to these kind of coagulation equations. These results will be presented next. 


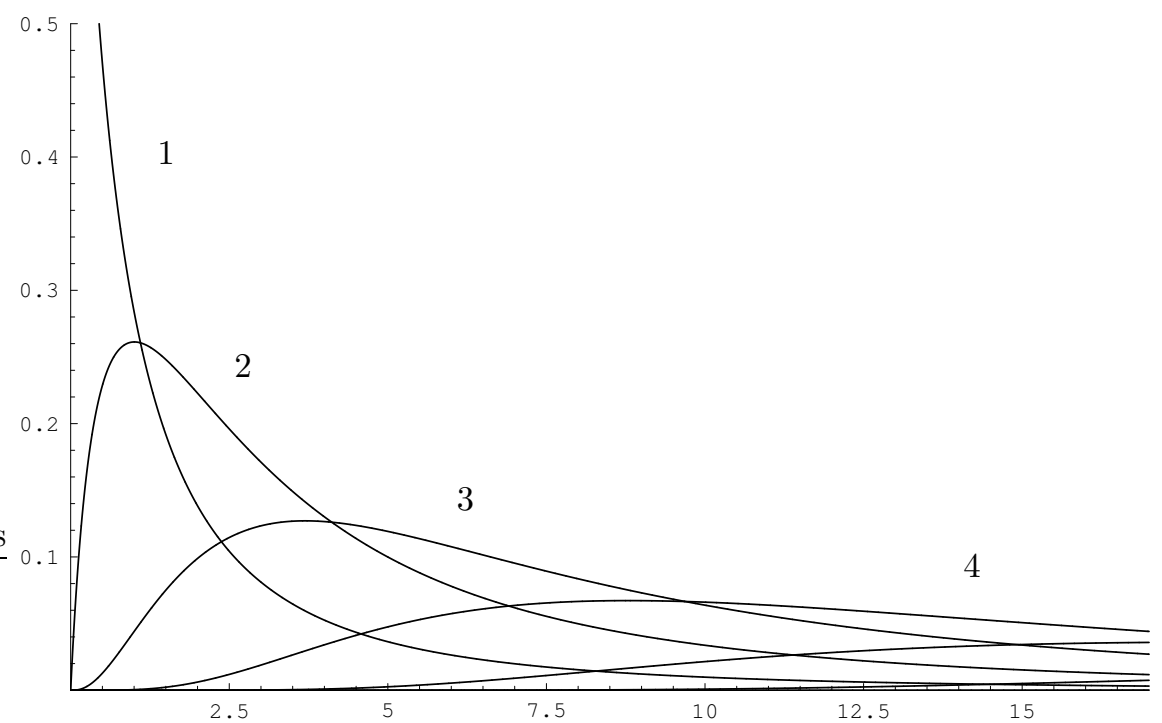

Figure 6: Numerical solution of a truncation of (67) of dimension seven, with monomeric initial condition. The plots of $N_{p}$ for $p$ from 1 to 6 are shown.

\subsubsection{On Similarity Solutions}

In order to seek a similarity solution, we first replace the system of nonlinear differential-difference equations by a single nonlinear partial differential equation. This is performed by taking Taylor series of the difference terms and keeping only the most significant terms in the large $p$ limit. Thus we obtain

$$
\partial_{t} N=-2 N \partial_{p} N-N \int_{p}^{\infty} N(j, t) d j
$$

for the quantity $N(p, t)$. We now seek a similarity solution of the form

$$
N(p, t)=e^{-\gamma p} f(\eta), \quad \text { with } \quad \eta=t e^{-\gamma p},
$$

for the functions $N(p, t)$ with $p>1$. Inserting this ansatz into equation (68) we obtain the integro-differential equation

$$
f^{\prime}(\eta)=2 \gamma f(\eta)\left[f(\eta)+\eta f^{\prime}(\eta)\right]-\frac{f(\eta)}{\gamma \eta} \int_{0}^{\eta} f(\xi) d \xi
$$

Rearrangement and differentiation leads to the second-order ordinary differential equation

$$
f(\eta)=2 \gamma^{2} \frac{d}{d \eta}\left(\eta\left(f+\eta \frac{d f}{d \eta}\right)\right)-\gamma \frac{d}{d \eta}\left(\frac{\eta}{f} \frac{d f}{d \eta}\right)
$$


which can be converted into an autonomous second-order equation by the change of variables $\eta=e^{\zeta}, f(\eta)=e^{-\zeta} \phi(\zeta)$ yielding

$$
0=\frac{d^{2} \phi}{d \zeta^{2}}\left(2 \gamma^{2} \phi^{2}-\gamma \phi\right)+\gamma\left(\frac{d \phi}{d \zeta}\right)^{2}-\phi^{3}
$$

Standard phase plane techniques allow this equation to be analysed. The phase plane is illustrated in Figure 7 which shows the trajectories, including homoclinic connections to the origin $\left(\phi=0=\phi^{\prime}\right)$.

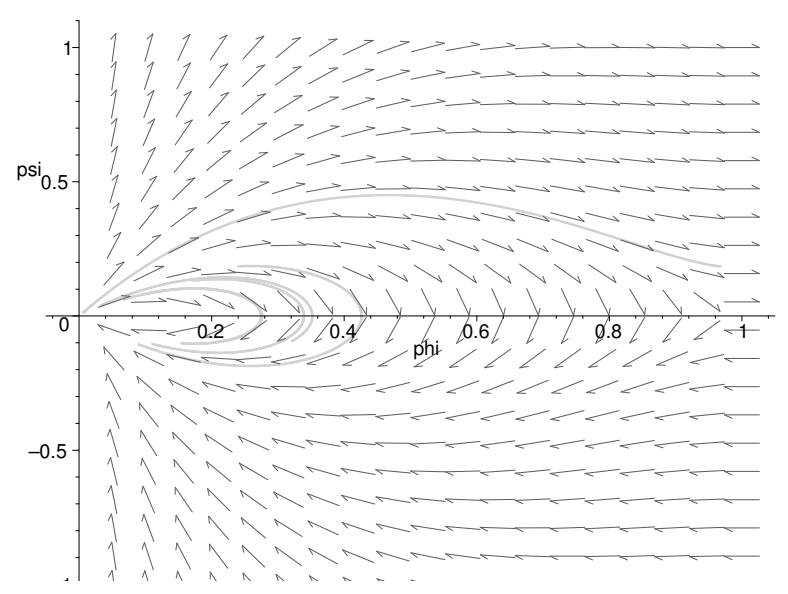

Figure 7: Phase plane $(\phi, \psi)$.

The analytic form of these trajectories can be found: by putting $\psi=\phi^{\prime}$ we obtain

$$
\frac{d \psi}{d \phi}=\frac{\gamma \psi^{2}-\phi^{3}}{\gamma \phi \psi(1-2 \gamma \phi)}
$$

which on integrating yields

$$
\left(\frac{d \phi}{d \zeta}\right)^{2}=\psi^{2}=\frac{2 \phi^{2}\left(\phi_{0}-\gamma \phi_{0}^{2}-\phi+\gamma \phi^{2}\right)}{\gamma(1-2 \gamma \phi)^{2}}
$$

where we have set the constant of integration so that at the maximum of $\phi(\zeta)$, where $\psi=\phi^{\prime}=0$, we have $\phi=\phi_{0}$. The above equation can then be integrated further to

$$
\pm \log \eta= \pm \zeta=\sqrt{\frac{\gamma}{2}} \int_{\eta f(\eta)}^{\phi_{0}} \frac{(1-2 \gamma \phi) d \phi}{\phi \sqrt{\left(\phi_{0}-\phi\right)\left(1-\gamma \phi_{0}-\gamma \phi\right)}}
$$

yielding parametric solutions for $(\eta(\zeta), f(\zeta))$. Solutions for $N_{p}(t)$ are then given by (69), and are illustrated in Figure 8 for a variety of choices of the parameter $\gamma$. 


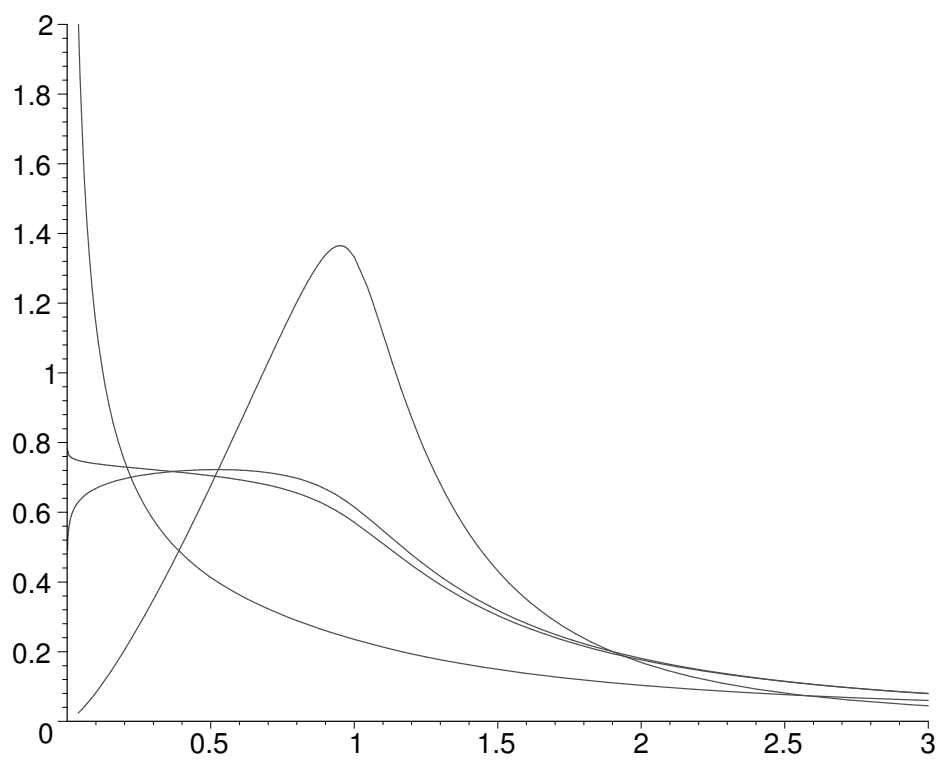

Figure 8: Plots of $f$ as a function of $\eta$ for several values of $\gamma$. Case $\gamma=0.3$ shows a clear maximum at $\eta=1, \gamma=0.65$ shows a plateau with a maximum at $\eta \approx 0.5, \gamma=0.7$ also has a plateau for $0<\eta<1$ but always has a negative gradient, and $\gamma=1.7$ which has a large negative gradient at small $\eta$. In all cases $\phi_{0}=0.4 / \gamma$.

\subsubsection{Intepretation of results}

The existence of a self-similar form for the functions $N_{p}=e^{-\gamma p} f(\eta)$ with $\eta=$ $t e^{-\gamma p}$ provides information about the positions or times of the maxima of $N_{p}(t)$, following the notation used earlier, these are defined by $t=\tau_{p}$. Since such points occur where $\dot{N}_{p}=0$, they correspond to the point where $f^{\prime}(\eta)=0$; we denote the $\eta$-value of such a point by $\eta=\eta_{c}$. Stationary points thus occur at $t_{p}=\eta_{c} e^{\gamma p}$. The amplitude of $N_{p}(t)$ at such a point is defined by $N_{p}^{\max }=N_{p}\left(\tau_{p}\right)$. The amplitude of the maxima are given by $N_{p}^{\max }=e^{-\gamma p} f_{c}$ where $f_{c}=f\left(\eta_{c}\right)$, which implies

$$
\frac{N_{p}^{\max }}{N_{p+1}^{\max }}=e^{\gamma}
$$

Raw numerical results of $N_{p}(t)$ against $t$ are shown in Figure 6; in Figure 9, the same data is replotted, with $t e^{-p \gamma}$ on the horizontal axis and $e^{p \gamma} N_{p}(t)$ on the vertical. Varying $\gamma$ so as to provide the best fit yields $\gamma=0.623$.

The one-dimensional family of similarity curves for $\gamma=0.623$ are displayed in Figure 10, where $\phi_{0}$ is varied to illustrate the range of curves. In general, the shape of the similarity solution varies with the parameters $\gamma$ and $\phi_{0}$, exhibitting a change from a single-humped function to a monotone curve at $\phi_{0} \gamma \approx 0.264$.

The shape of the similarity solution varies with the parameters $\gamma, \phi_{0}$, and 


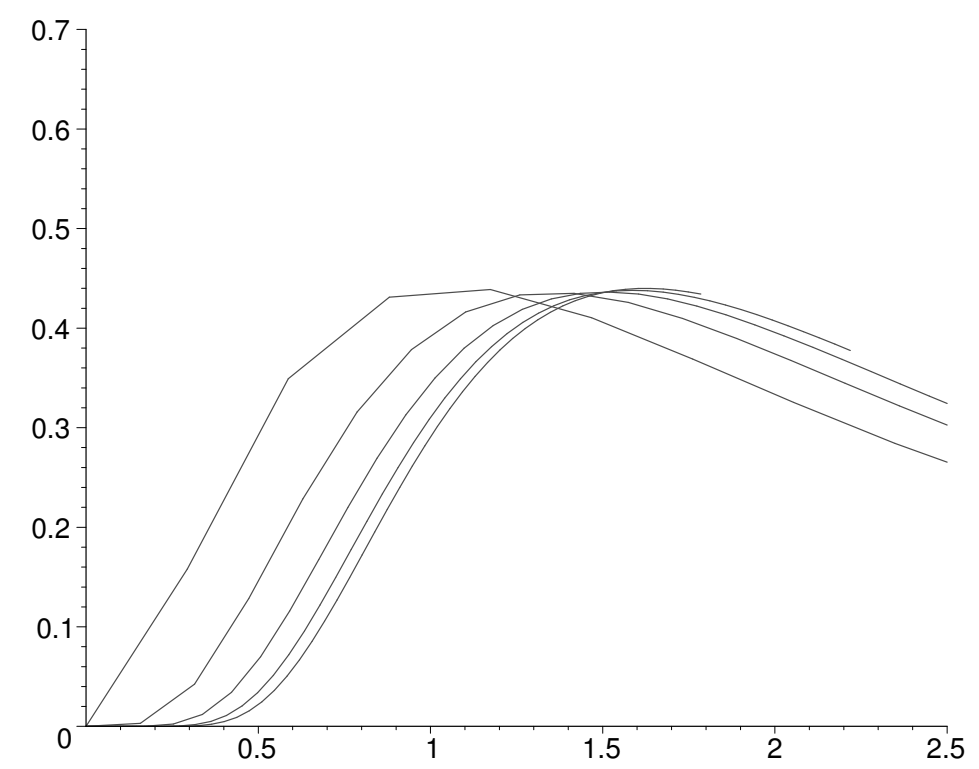

Figure 9: Scaled numerical solutions: $e^{-p \gamma} N_{p}\left(t / e^{\gamma p}\right)$ is plotted against $e^{-p \gamma} t$ for several values of $p$, with $\gamma$ fitted to give the best possible fit, which occurred when $\gamma=0.623$.

exhibits a change from being a single-humped function to a monotone curve at $\phi_{0} \gamma \approx 0.264$.

\section{Discussion}

As we stated in the Introduction, the objective of the present paper was to propose and analyse a system of ordinary differential equations describing the time evolution of a cluster system obeying Horton-Strahler rules. This kind of approach was first developed recently by Gabrielov, Newmann, and Turcotte in [11].

Our main objective was to obtain results concerning the long time behaviour of solutions.

Part of our goal was attained: Section 4 consists of the proofs that all solutions converge pointwise to zero: $c_{i, j}(t) \rightarrow 0$ as $t \rightarrow \infty$; and that the total number of clusters of a fixed order, $N_{i}(c(t))$, also converge to zero as $t \rightarrow \infty$. From a mathematical viewpoint the proof of the first of these results has the interesting feature of requiring the use of two families of Lyapunov functionals. An evolution inequality between the two allow us to deduce the result. The proof of the result concerning the evolution of the mesoscopic variables $N_{i}(c(t))$ requires the deduction of an evolution system for these quantities, from where evolutions inequalities used in the proof are obtained. 


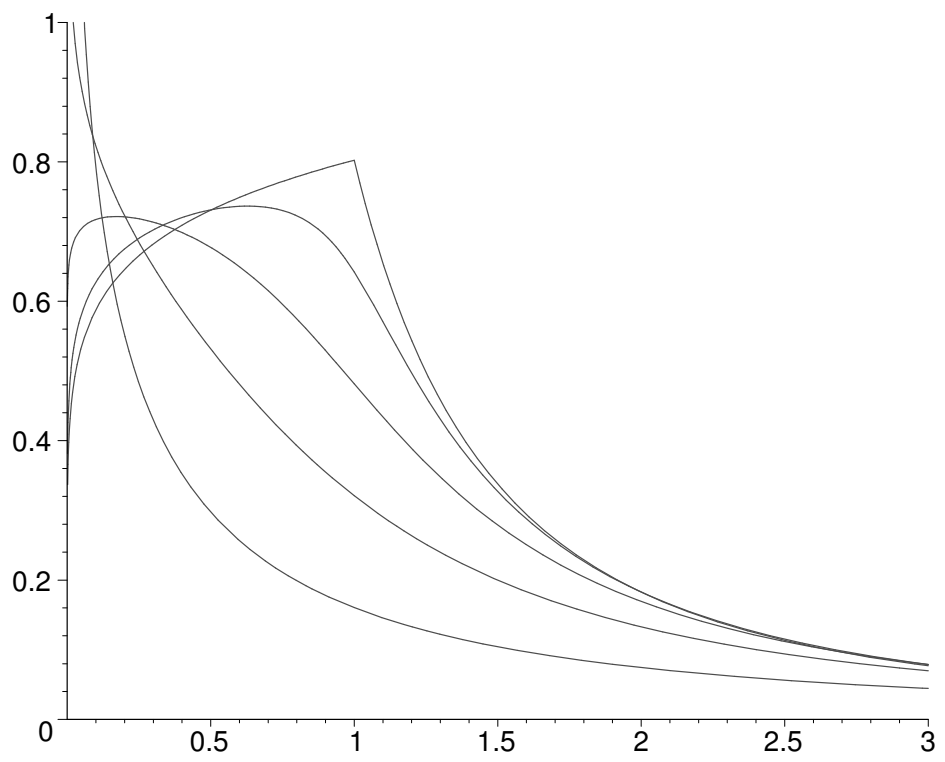

Figure 10: Solutions for $f(\eta)$ against $\eta$ for $\gamma=0.623$. In ascending order of values at $\eta=1$, the curves correspond to $\gamma \phi_{0}=0.1,0.2,0.3,0.4,0.5$.

Either the evolution system or the inequalities suggests symplified approximating systems of differential equations for the quantities $N_{i}$ whose behaviour should be easier to understand and analyse. In section 5 we have considered two of these simplified systems. The simplified systems allowed approximate solutions to be found. Two of these mesoscopic models have been analysed: firstly a local interaction model, and secondly a more complicated nonlocal model, which is expected to provide a more accurate approximation to the dynamics of the full system.

For the first we were able to prove a number of results concerning the shape of the solutions, namely the unimodality of solutions with monomeric initial data. These were also complemented with an asymptotic analysis concerning the long time profile of solutions. It is interesting to observe that some of the results reflect a kind of underlying structure of the equation for the $N_{p}(t)$ for large $p$ and $t$ : in these circunstances, approximating $p$ by a continuous variable, the local interaction system can be written, in first approximation, as the Burgers equation $\partial_{t} N+2 N \partial_{p} N=0$. It is interesting to observe that the assymptotic solution obtained in Section 5.1.2 is a solution of Burgers equation. Also, the fact that the larger the value of $p$, the longer the initial profile of $N_{p}(t)$ is almost identically zero (which is analitically reflected by Lemma 1) corresponds to the existence of a shock supported by the Burgers equation with a point source at the origin and a function with the behaviour of $N_{1}(t)$ as the boundary condition at $p=0$.

For the second approximating system a formal analysis yielded a similarity 
solution, which is expected to be an attractor in the large- $p$ and large- $t$ limits. Results of numerical simulations of the system agreed well with the similarity reduction. However comparison with physical observations was less convincing; these comparisons were based on the ratios of the amplitudes of successive maxima; in physical observations these are typically in the range three to five, for the model analysed here the ratio was approximately two. The reason for this discrepancy is possibly due to the form of rate coefficients chosen for the detailed study. The mesoscopic systems studied assumed $l_{j}=1$, in a future work we hope to analyse more general forms of rate coefficients, of the form $l_{j}=L j^{\lambda}$, where more general ratios are likely to be found, and hence the appropriate form of rate constants for modelling river networks, will also be determined.

\section{Acknowledgements}

We thank Jack Carr for helpful discussions. We also thank Philippe Laurençot some discussions about the local interaction system and Burger's equation, and Philippe Chassaing for pointing us some references about the use of HortonStrahler rules in Computer Science and in Combinatorics. A preliminary version of these results was presented by FPC at a meeting in the MFO, Germany, in August 2001. FPC thanks the support of FCT (Portugal) through program POCTI and project POCTI/32931/MAT/2000, and of Fundação Calouste Gulbenkian.

\section{References}

[1] P.B. DubovskiI, Mathematical Theory of Coagulation, Research Institute of Mathematics/Global Analysis Research Center, Lecture Notes Series No. 23, Seoul National University, Seoul, 1994.

[2] P. Coveney, J.A.D. Wattis, Becker-Döring model of self-reproduction vesicles, J. Chem. Soc., Faraday Trans., 94, 233-246 (1998).

[3] J.A.D. WAtTis, A Becker-Döring model of competitive nucleation, $J$. Phys. A: Math. Gen., 32, 8755-8784 (1999).

[4] R. Dunwell, The Becker-Döring Cluster Equations, PhD Thesis, HeriotWatt University, Edinburgh, 1997.

[5] R. Horton, Erosional development of streams and their drainage basins; hydrophysical approach to quantitative morphology, Bull. Geol. Soc. Amer., 56, 275-370 (1945).

[6] A.N. Strahler, Hypsometric (area-altitude) analysis of erosional topography, Bul. Geol. Soc. Amer., 63, 1117-1142 (1952).

[7] L.B. Leopold, M.G. Wolman, J.P.Miller, Fluvial Processes in Geomorphology, W.H. Freeman, San Francisco, 1970. 
[8] A.E. Scheidegger, Theoretical Geomorphology, 2nd revised Ed., Allen \& Unwin/Springer-Verlag, London/Berlin, 1970.

[9] A.P. Ershov, On programming of arithmetic operations, Comm. of the $A C M, \mathbf{1},(8) 3-6$ (1958).

[10] X.G. Viennot, Trees everywhere, in A. Arnold (Ed.), CAAP'90: 15th Colloquium on trees in algebra and programming, Copenhagen, Denmark, May 1990; pp 18-41, Lecture Notes in Computer Science 431, SpringerVerlag, Heidelberg, 1990.

[11] A. Gabrielov, W.I. Newman, D.L. Turcotte, Exactly soluble hierarchical clustering model: inverse cascades, self-similarity, and scaling, Phys. Rev. E, 60, (5) 5293-5300 (1999).

[12] J. Carr, F.P. DA Costa, J.A.D. Wattis, (work in progress).

[13] P. Coveney, J.A.D. Wattis, Cluster renormalization in the BeckerDöring equations, J. Phys. A: Math. Gen., 32, 7145-7152 (1999).

[14] F.P. DA Costa, A finite dimensional dynamical model for gelation in coagulation processes, J. Nonlinear Sci., 8, 619-653 (1998).

[15] Ph. LaurençOt, Global solutions to the discrete coagulation equations, Mathematika, 46, 433-442 (1999).

[16] J.M. BALL, J. CARR, The discrete coagulation-fragmentation equations: existence, uniqueness, and density conservation, J. Stat. Phys., 61, 203-234 (1990).

[17] F.P. DA Costa, Existence and uniqueness of density conserving solutions to the coagulation-fragmentation equations with strong fragmentation, $J$. Math. Anal. Appl., 192, 892-914 (1995).

[18] J. CARr, F.P. DA Costa, Asymptotic behavior of solutions to the coagulation-fragmentation equations. II. Weak fragmentation, J. Stat. Phys., 77, 89-123 (1994).

[19] J.M. Ball, J. Carr, O. Penrose, The Becker-Döring cluster equations: basic properties and asymptotic behaviour of solutions, Commun. Math. Phys., 104, 657-692 (1986).

[20] W.H. White, A global existence theorem for Smoluchowski's coagulation equations, Proc. Amer. Math. Soc., 80, 273-276 (1980).

[21] F. Leyvraz, H.R. Tschudi, Singularities in the kinetics of coagulation processes, J. Phys. A: Math. Gen., 14, 3389-3405 (1981).

[22] M. GRinfeld, Notes on fracture, (unpublished notes). 
[23] A.N. Strahler, Quantitative analysis of watershed geomorphology, Trans. Amer. Geophy. Union, 38, 913-920 (1957).

Instituto Superior TÉCNico UNIVERSiTy OF STRATHCLYDE UNIVERSITY OF NOTTINGHAM

(November 29, 2001) 